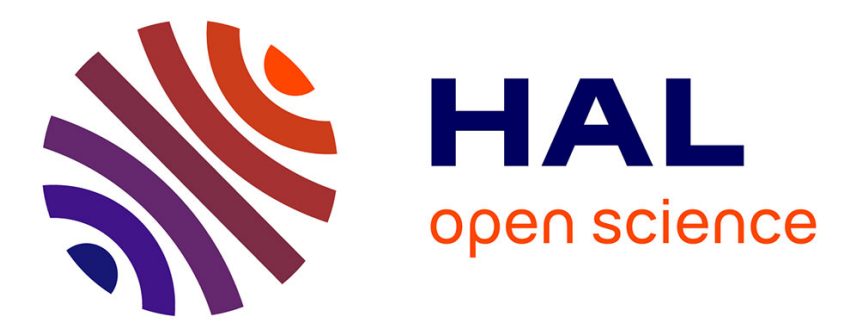

\title{
PGD-Based Computational Vademecum for Efficient Design, Optimization and Control
}

Francisco Chinesta, Adrien Leygue, Felipe Bordeu, Elías Cueto, David

Gonzalez, Amine Ammar, Antonio Huerta

\section{- To cite this version:}

Francisco Chinesta, Adrien Leygue, Felipe Bordeu, Elías Cueto, David Gonzalez, et al.. PGD-Based Computational Vademecum for Efficient Design, Optimization and Control. Archives of Computational Methods in Engineering, 2013, 20 (1), pp.31 - 59. 10.1007/s11831-013-9080-x . hal-01515083v2

\section{HAL Id: hal-01515083 \\ https://hal.science/hal-01515083v2}

Submitted on 2 May 2017

HAL is a multi-disciplinary open access archive for the deposit and dissemination of scientific research documents, whether they are published or not. The documents may come from teaching and research institutions in France or abroad, or from public or private research centers.
L'archive ouverte pluridisciplinaire HAL, est destinée au dépôt et à la diffusion de documents scientifiques de niveau recherche, publiés ou non, émanant des établissements d'enseignement et de recherche français ou étrangers, des laboratoires publics ou privés. 


\title{
PGD-based Computational Vademecum for efficient design, optimization and control
}

\author{
F. Chinesta ${ }^{1}$, A. Leygue ${ }^{2}$, F. Bordeu ${ }^{2}$, E. \\ Cueto $^{3}$, D. Gonzalez ${ }^{3}$, A. Ammar ${ }^{4}$, A. Huerta ${ }^{5}$
}

\begin{abstract}
In this paper we are addressing a new paradigm in the field of simulationbased engineering sciences (SBES) to face the challenges posed by current ICT technologies. Despite the impressive progress attained by simulation capabilities and techniques, some challenging problems remain today intractable. These problems, that are common to many branches of science and engineering, are of different nature. Among them, we can cite those related to high-dimensional problems, which do not admit mesh-based approaches due to the exponential increase of degrees of freedom. We developed in recent years a novel technique, called Proper Generalized Decomposition (PGD). It is based on the assumption of a separated form of the unknown field and it
\end{abstract}

This work has been partially supported by the Spanish Ministry of Science and Competitiveness, through grants number CICYT-DPI2011-27778-C02-01/02. Professor Chinesta is also supported by the Institut Universitaire de France.

\author{
F. Chinesta \\ EADS Foundation Chair "Advanced Computational Manufacturing Processes" \\ GEM, UMR CNRS - Centrale Nantes \\ Institut Universtaire de France \\ 1 rue de la Noe, BP 92101, F-44321 Nantes cedex 3, France \\ E-mail: Francisco.Chinesta@ec-nantes.fr \\ A. Leygue \& F. Boredeu \\ EADS Foundation Chair "Advanced Computational Manufacturing Processes" \\ GEM, UMR CNRS - Centrale Nantes \\ 1 rue de la Noe, BP 92101, F-44321 Nantes cedex 3, France \\ E-mail: \{Adrien.Leygue,Felipe.Bordeu\}@ec-nantes.fr \\ E. Cueto \& D. Gonzalez \\ I3A, Universidad de Zaragoza \\ Maria de Luna s/n, 50018 Zaragoza, Spain \\ E-mail: \{ecueto,gonzal\}@unizar.es \\ A. Ammar \\ Arts et Métiers ParisTech \\ 2 Boulevard du Ronceray, BP 93525, F-49035 Angers cedex 01, France \\ E-mail: Amine.AMMAR@ensam.eu \\ A. Huerta \\ Laboratori de Calcul Numeric \\ Universidad Politecnica de Cataluña \\ Jordi Girona, 1 Campus Nord, C2, E-08034 Barcelona, Spain \\ E-mail: antonio.huerta@upc.es
}


has demonstrated its capabilities in dealing with high-dimensional problems overcoming the strong limitations of classical approaches. But the main opportunity given by this technique is that it allows for a completely new approach for classic problems, not necessarily high dimensional. Many challenging problems can be efficiently cast into a multidimensional framework and this opens new possibilities to solve old and new problems with strategies not envisioned until now. For instance, parameters in a model can be set as additional extra-coordinates of the model. In a PGD framework, the resulting model is solved once for life, in order to obtain a general solution that includes all the solutions for every possible value of the parameters, that is, a sort of virtual chart. Under this rationale, optimization of complex problems, uncertainty quantification, simulation-based control and real-time simulation are now at hand, even in highly complex scenarios, by combining an off-line stage in which the general PGD solution, the virtual chart, is computed, and an on-line phase in which, even on deployed, handheld, platforms such as smartphones or tablets, real-time response is obtained as a result of our queries.

\section{Introduction}

\subsection{Motivation}

Six unique initiatives have been recently selected (and funded with 100 millions of euros per year) by the European Research Council based solely on their potential for realizing scientific breakthroughs and influencing Europe's social and industrial challenges, including health. Their aim will then be to deliver major breakthroughs in information and communication technologies (ICT), with the potential to provide solutions to some of society's biggest challenges. Despite being different there is a common ingredient to all of them, which is to emphasize the necessity of making use of advanced simulation-driven sciences and engineering, as will be highlighted below. The six contenders, from which the two flagship initiatives will be selected, are: [1]

1. Guardian Angels for a Smarter Life [2]: a project aimed at developing tiny devices without batteries that act like thinking and autonomous personal assistants, providing information and communication technologies to assist people in all sorts of complex situations delivering features and characteristics that go well beyond human capabilities.

2. The Human Brain Project [3] whose goal is to understand the way the human brain works. The long-term goal of the Human Brain Project is to build the informatics, modeling, and supercomputing technologies that are needed to simulate and understand the human brain.

3. IT Future of Medicine [4] proposes a data-driven, individualized medicine of the future, based on the molecular/physiological/anatomical data from individual patients. The project outcomes will enable data-driven real-time calculation of health, disease, therapy and its effects for individual patients.

4. Robot Companions for Citizens [5]: a project devoted to developing soft-skinned and intelligent robots with highly developed perceptive, cognitive and emotional skills. Robot Companions for Citizens will be based on the novel solid articulated structures with flexible properties displaying soft behavior, haptic devices and simulation based real time control in deployed systems. These companions will also 
have new levels of perceptual, cognitive and emotive capabilities and be aware of their physical and social surroundings and respond accordingly.

5. FuturICT Knowledge Accelerator and Crisis-Relief System [6]: What if global scale computing facilities were available that could analyze most of the data available in the world? What insights could scientists gain about the way society functions? What new laws of nature would be revealed? Could society discover a more sustainable way of living? ICT (Information and Communication Technology) can analyze vast amounts of data and complex situations so as to better predict natural disasters, or manage and respond to man-made disasters that cross national borders or continents.

6. Graphene Science and technology for ICT and beyond [7]: Graphene is a new substance developed by atomic and molecular scale manipulation that could replace silicon as the wonder material of the 21st century. This aims to explore revolutionary potentials, in terms of both conventional as well as radically new fields of Information and Communication Technologies applications.

It is now well known [3] that the human brain consumes 4 watts for performing some tasks that today's computers will require the power of several nuclear plants. It is then clear that our computers and algorithms for addressing the models encountered in science and engineering are definitively suboptimal. The above six flagship projects share some key aspects related to efficient computational sciences. It is expected that these projects will reach a certain number of breakthroughs, but all of them will face important limitations of today's computer capabilities and, notably, simulation techniques.

All these society needs require fast and accurate solutions, in general data-driven, of very complex models, involving an unimaginable amount of information, in most cases in real time and on deployed platforms. Up to now, the solution of complex models, preferably fast and accurate, is addressed by using high performance computing and hyper powerful computing platforms. Obviously the consecution of the above "dreams" will require as much as computational power (supercomputing) as possible, and consequently, advances in hardware and software for high-performance computing will be necessary. But at the same time, there is a need for a new generation simulation techniques, beyond high-performance computing or nowadays approaches (most of them proposed 40 years ago), to simply improve efficiency or to allow getting results when other alternatives fail in the above challenging scenarios.

All the above challenging problems are data-driven. The importance of Dynamic Data-Driven Application Systems -DDDAS - in the forthcoming decades has been already noticed by the NSF Blue Ribbon Panel on Simulation Based Engineering Sciences report, that in 2006 included DDDAS as one of the five core issues or challenges in the field for the next decade (together with multi-scale simulation, model validation and verification, handling large data and visualization). This panel concluded that "Dynamic data-driven application systems will rewrite the book on the validation and verification of computer predictions" and that "research is needed to effectively use and integrate data-intensive computing systems, ubiquitous sensors and high-resolution detectors, imaging devices, and other data-gathering storage and distribution devices, and to develop methodologies and theoretical frameworks for their integration into simulation systems" [61] [32] [62]. Moreover, the NSF believes that "... The DDDAS community needs to reach a critical mass both in terms of numbers of investigators, and in terms of the depth, breadth and maturity of constituent technologies ..." [61]. 


\subsection{Nowadays computational issues}

Today many problems in science and engineering remain intractable, in spite of the impressive progresses attained in modeling, numerical analysis, discretization techniques and computer science during the last decade, because their numerical complexity, or the restrictions imposed by different requirements (real-time on deployed platforms, for instance) make them unaffordable for today's technologies.

We can enumerate different challenging scenarios for efficient numerical simulations:

- The first one concerns models that are defined in high dimensional spaces, usually encountered in quantum chemistry describing the structure and mechanics of materials [11] [25], the kinetic theory description of complex materials [20] [48], social dynamics and economic systems, vehicular traffic flow phenomena, complex biological systems involving mutation and immune competition, crowds and swarms encountered in congested and panic flows, among many other unimaginable possibilities (see [18] and the references therein); the chemical modeling in too dilute systems where the concept of concentration cannot be used, that results in the so-called chemical master equation governing for example cell signaling and other phenomena in molecular biology [16].

Models defined in high dimensional spaces suffer the so-called curse of dimensionality. If one proceeds to the solution of a model defined in a space of dimension $d$ by using a standard mesh based discretization technique, where $\mathcal{M}$ nodes are used for discretizing each space coordinate, the resulting number of nodes reaches the astronomical value of $\mathcal{M}^{d}$. With $\mathcal{M} \approx 10^{3}$ (a very coarse description in practice) and $d \approx 30$ (a very simple model) the numerical complexity results $10^{90}$. It is important to recall that $10^{80}$ is the presumed number of elementary particles in the universe!.

Traditionally, high dimensional models were addressed by using stochastic simulations. However these techniques have their own challenges: variance reduction is always an issue and the construction of distribution functions in high dimensional spaces remains in most cases unaffordable. It is also quite difficult within the stochastic framework to implement parametric or sensitivity analysis that go beyond the brute force approach of computing a large number of expensive, individual simulations.

- Online control can be carried out following different approaches. The most common one consists in considering systems as a black box whose behavior is modeled by a transfer function relating certain inputs to certain outputs. This modeling that may seem poor has as main advantage the possibility of proceeding rapidly due to its simplicity. This compromise between accuracy and rapidity was often used in the past and this pragmatic approach has allowed us to control processes and to optimize them, once the transfer function modeling the system is established.

The establishment of such goal-oriented transfer function is the trickiest point. For this purpose, it is possible to proceed from a sometimes overly simplified physical model or directly from experiments (allowing us to extract a phenomenological goaloriented transfer function) or from a well-balanced mixture of both approaches. In all cases, the resulting modeling can only be applied within the framework that served to derive it. However, on one hand, the fine description of systems requires a sufficiently detailed description of them and, in that case, traditional goal-oriented simplified modeling becomes inapplicable. On the other hand, actual physical mod- 
els result, in general, in complex mathematical objects, non-linear and strongly coupled partial differential equations. Such mathematical objects are representing physical reality up to a certain degree of accuracy. However, the available numerical tools capable of solving these complex models require the use of powerful computers that can require hours, days and weeks to solve them. Known as numerical simulation, its output solution is very rich but it seems inapplicable for control purposes that require fast responses, often in real-time.

Until now, numerical simulation has been used offline but in some cases it allows us to define simplified models (with their inherent limitations and drawbacks) running in real-time that could be used online but such simplified modeling has the previously quoted drawbacks.

- Many problems in parametric modeling, inverse identification, and process or shape optimization, usually require, when approached with standard techniques, the direct computation of a very large number of solutions of the concerned model for particular values of the problem parameters. When the number of parameters increases such a procedure becomes inapplicable.

- Traditionally, Simulation-based Engineering Sciences - SBES - relied on the use of static data inputs to perform the simulations. These data could be parameters of the model(s) or boundary conditions. The word static is intended to mean here that these data could not be modified during the simulation. A new paradigm in the field of Applied Sciences and Engineering has emerged in the last decade. Dynamic Data-Driven Application Systems (DDDAS) constitute nowadays one of the most challenging applications of simulation-based Engineering Sciences. By DDDAS we mean a set of techniques that allow the linkage of simulation tools with measurement devices for real-time control of simulations. DDDAS entails the ability to dynamically incorporate additional data into an executing application, and in reverse, the ability of an application to dynamically steer the measurement process.

In this context, real time simulators are needed in many applications. One of the most challenging situations is that of haptic devices, where forces must be translated to the peripheral device at a rate of $500 \mathrm{~Hz}$. Control, malfunctioning identification and reconfiguration of malfunctioning systems also need to run in real time. All these problems can be seen as typical examples of DDDAS.

- Augmented reality is another area in which efficient (fast and accurate) simulation is urgently needed. The idea is supplying in real time appropriate information to the reality perceived by the user. Augmented reality could be an excellent tool in many branches of science and engineering. In this context, light computing platforms are appealing alternatives to heavy computing platforms that in general are expensive and whose use requires technical knowledge.

- Inevitable uncertainty. In science and engineering, in its widest sense, it now seems obvious that there are many causes of variability. The introduction of such variability, randomness and uncertainty is a priority for the next decade. Although it was a priority in the preceding decade, the practical progress attained seems fairly weak.

While the previous list is by no means exhaustive, it includes a set of problems with no apparent relationship between them that can however be treated in a unified manner as will be shown in what follows. Their common ingredient is our lack of capabilities (or knowledge) to solve them numerically in a direct, traditional way. 


\section{Charts in science and engineering}

The human being throughout the history developed several facilities for giving fast responses to a variety of questions. Thus, abaci were used 2700 years B.C. in Mesopotamia. This abacus was a sort of counting frame primarily used for performing arithmetic calculations. We associate this abacus to a bamboo frame with beads sliding on wires, however, originally they were beans or stones moved in grooves in sand or on tablets of wood, stone, or metal. The abacus was in use centuries before the adoption of the written modern numeral system and is still widely used by raders. There are many variants, the Mesopotamian abacus, the Egyptian, Persian, Greek, Roman, Chinese, Indian, Japanese, Korean, native American, Russian, etc.

However, the initial arithmetic needs were rapidly complemented with more complex representations. We are considering some few variants:

- Charts appeared for graphical representation of data with multiple meanings. However, there are common features that provide the chart with its ability to extract meaning from data. In general a chart is graphical, containing very little text, since humans infer meaning from pictures quicker than from text. A particular variant of charts in the Nomogram.

- Nomography, is the graphical representation of mathematical relationships or laws. It is an area of practical and theoretical mathematics invented in 1880 by Philbert Maurice d'Ocagne and used extensively for many years to provide engineers with fast graphical calculations of complicated formulas to a practical precision. Thus, a nomogram can be considered as a graphical calculating device. There are thousands of examples on the use of nomograms in all the fields of sciences and engineering.

The former facilities allowed for fast calculations and data manipulations. Nomograms can be easily constructed when the mathematical relationships that they express are purely algebraic, eventually non-linear. In those cases it was easy to represent some outputs as a function of some inputs. The calculation of these data representations was performed off-line and then used on-line in many branches of engineering sciences for design and optimization.

However, the former procedures fail when addressing more complex scenarios. Thus, sometimes engineers manipulate not properly understood physics and in that case the construction of nomograms based on a too coarse modelling could be dangerous. In that cases one could proceed by making several experiments from which defining a sort of experiment-based nomogram. In other cases the mathematical object to be manipulated consists of a system of complex coupled non-linear partial differential equations, whose solution for each possible combination of the values of the parameters that it involves is simply unimaginable for the nowadays computational availabilities. In these cases experiments or expensive computational solutions are performed for some possible states of the system, from which a simplified model linking the inputs to the outputs of interest is elaborated. These simplified models have different names: surrogate models, metamodels, response surface methodologies, ... Other associated tricky questions are the one that concerns the best sampling strategy (Latin hypercube, ...) and also the one concerning the appropriate interpolation techniques for estimating the response at an unmeasured position from observed values at surrounding locations. Many possibilities exist, being Kriging one of the most widely used for interpolating data. 
All these techniques allow defining a sort of numerical or graphical charts. However, we must accept a certain inevitable inaccuracy when estimating solutions from the available data. It is the price to pay if neither experimental measurements nor numerical solutions of the fine but expensive model are achievable for each possible scenario.

Recently model order reduction opened new possibilities. First, proper orthogonal decompositions (POD) allows extracting the most significant characteristic of the solution, that can be then applied for solving models slightly different to the ones that served to defined the reduced approximation bases. There is an extensive literature. The interested readers can reefer to [63] [54] [19] [24] [39] [9] [56] [57] [58] [59] [12] [70] and the numerous references therein. The extraction of the reduced basis is the tricky point when using POD-based model order reduction, as well its adaptivity when addressing scenarios far from the ones considered when constructing the reduced basis [68] [69]. Another issue lies in the error control, and its connection with verification and validation.

The calculation of the reduced basis is not unique. There are many alternatives. Some ones introduce some improvements on the POD methodology, as is the case of the Goal Oriented Model Constrained Optimization approach (see [23] and the references therein) or the modal identification method (see [36] and the references therein). The Branch Eigenmodes Reduction Method combined with the amalgam method is another appealing constructor of reduced bases [73].

Another family of model reduction techniques lies in the used of reduced basis constructed by combining a greedy algorithm and a priori error indicator. It needs for some amount off-line work but then the reduced basis can be used on-line for solving different models with a perfect control of the solution accuracy because the availability of error bounds. When the error is inadmissible, the reduced basis can be enriched by invoking again the same greedy algorithm. The interested readers can refer to [52] [53] [72] [67] and the references therein. The main drawback of such an approach is the amount of data that must be computed, stored and then manipulated.

Separated representations were introduced in the 80 s by Pierre Ladeveze that proposed a space-time separated representation of transient solutions involved in strongly non-linear models, defining a non-incremental integration procedure. The interested reader can refer to the numerous Ladeveze's works [40] [41] [42] [43] [44] [45] [64] [55]. Later, separated representations were employed in the context of stochastic modelling [60] as well as for solving multidimensional models suffering the so-called curse of dimensionality, some of them never solved before [8]. The techniques making use of separated representations computed on the fly were called Proper Generalized Decompositions - PGD -.

PGD constitutes an efficient multidimensional solver that allows introducing model parameters (boundary conditions, initial conditions, geometrical parameters, material and process parameters ...) as extra-coordinates. Then by solving only once and off-line the resulting multidimensional model we have access to the parametric solution that can be viewed as a sort of metamodel or virtual chart than can be then used on-line.

In what follows, we are describing within the PGD approach the way of introducing extra-coordinates of different nature. Later, we will prove the potentiality of such an approach for the efficient solution of a variety of problems. 


\subsection{PGD at a glance}

Consider a problem defined in a space of dimension $d$ for the unknown field $u\left(x_{1}, \cdots, x_{d}\right)$. Here, the coordinates $x_{i}$ denote any usual coordinate (scalar or vectorial) related to physical space, time, or conformation space in microscopic descriptions [8] [11], for example, but they could also include, as we illustrate later, problem parameters such as boundary conditions or material parameters. We seek a solution for $\left(x_{1}, \cdots, x_{d}\right) \in \Omega_{1} \times \cdots \times \Omega_{d}$.

The PGD yields an approximate solution in the separated form:

$$
u\left(x_{1}, \cdots, x_{d}\right) \approx \sum_{i=1}^{N} X_{i}^{1}\left(x_{1}\right) \cdot \ldots \cdot X_{i}^{d}\left(x_{d}\right)=\sum_{i=1}^{N} \prod_{j=1}^{d} X_{i}^{j}\left(x_{j}\right)
$$

The PGD approximation is thus a sum of $N$ functional products involving each a number $d$ of functions $X_{i}^{j}\left(x_{j}\right)$ that are unknown a priori. It is constructed by successive enrichment, whereby each functional product is determined in sequence. At a particular enrichment step $n+1$, the functions $X_{i}^{j}\left(x_{j}\right)$ are known for $i \leq n$ from the previous steps, and one must compute the new product involving the $d$ unknown functions $X_{n+1}^{j}\left(x_{j}\right)$. This is achieved by invoking the weak form of the problem under consideration. The resulting problem is non-linear, which implies that iterations are needed at each enrichment step. A low-dimensional problem can thus be defined in $\Omega_{j}$ for each of the $d$ functions $X_{n+1}^{j}\left(x_{j}\right)$.

If $\mathcal{M}$ nodes are used to discretize each coordinate, the total number of PGD unknowns is $N \cdot \mathcal{M} \cdot d$ instead of the $\mathcal{M}^{d}$ degrees of freedom involved in standard meshbased discretizations. We will come back later to the issues related to the convergence and optimality of the separated representations.

\subsection{Parametric solutions based virtual charts}

In the case of a field depending on the physical space $\mathbf{x} \in \Omega_{x} \subset \mathcal{R}^{3}$, the time $t \in \mathcal{I}_{t} \subset \mathcal{R}$ and $Q$ parameters $p^{1}, \cdots, p^{Q}, p^{j} \in \Omega_{p^{j}}, j=1, \cdots, Q$, the solution is sought under the separated form

$$
u\left(\mathbf{x}, t, p^{1}, \cdots, p^{Q}\right) \approx \sum_{i=1}^{N} X_{i}(\mathbf{x}) \cdot T_{i}(t) \cdot \prod_{j=1}^{Q} P_{i}^{j}\left(p^{j}\right)
$$

As soon as this solution is available, after solving the multidimensional model within the PGD framework, we can have access to any possible solution. In fact the representation (2) implies an approximation of each function. Thus the space functions $X_{i}(\mathbf{x})$ could be approximated for example by using a finite element interpolation that implies knowing the value of each space function $X_{i}(\mathbf{x})$ at the $\mathcal{M}_{x}$ nodes $\mathbf{x}_{k}\left(k=1, \cdots, \mathcal{M}_{x}\right)$ of the mesh used for approximating them, i.e. $X_{i}\left(\mathbf{x}_{k}\right)$. The functions $T_{i}(t)$ depending on time will be expressed from the values of those functions at $\mathcal{M}_{t}$ time instants $t_{l}$ $\left(l=1, \cdots, \mathcal{M}_{t}\right)$, i.e. $T_{i}\left(t_{l}\right)$. Finally, the functions depending on the different parameters $P_{i}^{j}\left(p^{j}\right)$ will be expressed from the values of those functions at $\mathcal{M}_{p^{j}}$ values of each parameter $p_{r}^{j}\left(r=1, \cdots, \mathcal{M}_{p^{j}}\right)$, i.e. $P_{i}^{j}\left(p_{r}^{j}\right)$. Thus, the solution consists of $N$ vectors of size $\mathcal{M}_{x}$ that contain the discrete representation of functions $X_{i}(\mathbf{x}), N$ vectors of 
size $\mathcal{M}_{t}$ that contain the discrete representation of functions $T_{i}(t)$ and $N$ vectors of size $\mathcal{M}_{p^{j}}$ that contain the discrete representation of functions $P_{i}^{j}\left(p^{j}\right), j=1, \cdots, Q$.

If we imagine that $\mathcal{M}_{x}=\mathcal{M}_{t}=\mathcal{M}_{p^{1}}=\cdots=\mathcal{M}_{p^{Q}}=\mathcal{M}$, then the solution representation involves $N \cdot(Q+2)$ vectors of size $\mathcal{M}$, that is $N \cdot(Q+2) \cdot \mathcal{M}$ values instead of the $\mathcal{M}^{2+Q}$ involved by an equivalent mesh. If for a while we imagine $Q=10$, $\mathcal{M}=100$ and $N=10$ the separated representation will involve $10^{4}$ values instead of $10^{20}$ involved in a hypothetical equivalent mesh.

Thus, the PGD solver allowing the construction of the separated representation (2) can be viewed as a solver that constructs on the fly a compressed representation of the model solution. In the case of $2 \mathrm{D}$ models, involving two generic coordinates $c_{1}$ and $c_{2}$ and symmetric and positive definite differential operators, the separated representation constructed by the PGD solver reads:

$$
u\left(c_{1}, c_{2}\right) \approx \sum_{i=1}^{N} C_{i}^{1}\left(c_{1}\right) \cdot C_{i}^{2}\left(c_{2}\right)
$$

where the number of modes $N$ corresponds to the one involved by the best separated representation of the model solution $u\left(c_{1}, c_{2}\right)$ obtained by applying on it a singular value decomposition -SVD -. In higher dimensions, the higher-order SVD counterpart, the so-called High Order SVD (HOSVD) is no more optimal. In any case the PGD constructor can be viewed as a sort of "a priori" HOSVD. Some mathematical results can be found in [13] [49] and the review [29] and the references therein.

When the differential operator involved in the model is non symmetric, the separated representation obtained by applying any of the nowadays available PGD solvers is suboptimal, that is, the number of terms in the finite sum $N$ is higher that the number of modes that the SVD (in 2D) or its higher order counterpart would require when performing an "a posteriori" decomposition of the model solution.

In any case, even when PGD calculates suboptimal separated representations, at least it allows calculating solutions of highly multidimensional models that cannot be solved by using more experienced mesh-based discretizations. Thus the possibility of solving a problem, even when its solution representation involves sometimes more terms than strictly needed, is in any case a real success. Moreover, in general the solution of models involving many parameters as extra-coordinates is performed only once and off-line, and then it is particularized on-line. In these cases the optimality issue is not crucial. However, when the solution involves too many terms, with respect to an hypothetical optimal representation, i.e. $N \gg N^{o p t}$, one can proceed to an "a posteriori" data compression, in order to alleviate as much as possible post-processing, that is vital when real time responses are envisaged. For this purpose, we assume that the suboptimal solution, calculated from the differential model by applying a PGD solver, reads

$$
u\left(x_{1}, \cdots, x_{d}\right) \approx \sum_{i=1}^{N} \prod_{j=1}^{d} X_{i}^{j}\left(x_{j}\right)
$$

and we look for an enhanced representation $u^{e n h}\left(x_{1}, \cdots x_{d}\right)$

$$
u^{e n h}\left(x_{1}, \cdots, x_{d}\right) \approx \sum_{i=1}^{\tilde{N}} \prod_{j=1}^{d} \tilde{X}_{i}^{j}\left(x_{j}\right)
$$


that verifies

$$
u^{e n h}\left(x_{1}, \cdots, x_{d}\right)=u\left(x_{1}, \cdots, u_{d}\right)
$$

whose integral counterpart writes

$$
\int_{\Omega_{1} \times \cdots \times \Omega_{d}} u^{*} \cdot\left(u^{e n h}\left(x_{1}, \cdots, x_{d}\right)-u\left(x_{1}, \cdots u_{D}\right)\right) d x_{1} \cdots d x_{d}=0
$$

that is solved by invoking again the PGD solver.

$u^{e n h}\left(x_{1}, \cdots x_{d}\right)$ results a more compact representation, i.e. $\tilde{N} \leq N$, sometimes $\tilde{N} \ll N$. The problem (4)-(7) is equivalent to the application of a HOSVD on the suboptimal solution (4). This post-compression allows substantial storage savings, an important factor when using deployed devices, and also significant CPU time savings when manipulating data in post-processing tasks. In real time applications this postcompression can be decisive.

Until now, we presented the PGD as en efficient solver, however it can be also viewed as a model reduction strategy when computing only the most relevant terms of the separated representation. In that case an error is introduced, but because the few number of terms retained, storage and data post-processing can be efficiently performed.

In any case, as soon as a suboptimal or an enhanced separated representation is available, it can be viewed as a virtual chart, a metamodel, containing an unimaginable amount of information. For example, if the solution (2) applies for a model involving 10 parameters, all them considered as extra-coordinates, i.e. $Q=10$, and 10 discrete values are considered for describing each parametric dependency, i.e. $\mathcal{M}_{1}=\cdots=\mathcal{M}_{10}=$ 10 the solution (2) contains the information associated to $10^{10}$ possible scenarios. Obtaining an equivalent amount of information would require the solution of $10^{10}$ transient 3D problems. Now, from this extremely rich metamodel, one could obtain the solution for any possible scenario $u\left(\mathbf{x}, t, p^{1}, \cdots, p^{10}\right)$ from the parametric solution $(2)$.

\section{Parametric PGD based virtual charts: going beyond the nowadays computational limits}

In this section we revisit the computational issues enumerated in the first section from the perspective of parametric PGD based virtual charts described in the previous section. For this purpose, we are selecting some scenarios, that prove the potentiality of the proposed approach. We first expose the main ideas though simple academic examples based on the solution of the heat equation, and then we address in the next section more complex situations.

\subsection{Model parameters as extra-coordinates}

In this section, we illustrate the PGD by considering the following parametric heat transfer equation:

$$
\frac{\partial u}{\partial t}-k \cdot \Delta u-f=0
$$


with homogeneous initial and boundary conditions. The enforcement of non-homogeneous initial and boundary conditions was deeply treated in [37] and [26].

Here $(\mathbf{x}, t, k) \in \Omega \times \mathcal{I}_{t} \times \mathcal{I}_{k}$, with $\Omega \subset \mathcal{R}^{3}, \mathcal{I}_{t} \subset \mathcal{R}$ and $\mathcal{I}_{k} \subset \mathcal{R}$. For the sake of simplicity and without loss of generality the source term $f$ is assumed constant. The conductivity $k$ is viewed as a new coordinate defined in the interval $\mathcal{I}_{k}$. Thus, instead of solving the thermal model for different discrete values of the conductivity parameter, we wish to solve at once a more general problem, the price to pay being an increase of the problem dimensionality. However, as the complexity of the PGD scales only linearly (and not exponentially) with the space dimension, consideration of the conductivity as a new coordinate still allows one to efficiently obtain an accurate solution.

The weighted residual form related to Eq. (8) reads:

$$
\int_{\Omega \times \mathcal{I}_{t} \times \mathcal{I}_{k}} u^{*} \cdot\left(\frac{\partial u}{\partial t}-k \cdot \Delta u-f\right) d \mathbf{x} \cdot d t \cdot d k=0
$$

for all test functions $u^{*}$ selected in an appropriate functional space.

The PGD solution is sought in the form:

$$
u(\mathbf{x}, t, k) \approx \sum_{i=1}^{N} X_{i}(\mathbf{x}) \cdot T_{i}(t) \cdot K_{i}(k)
$$

At enrichment step $n$ of the PGD algorithm, the following approximation is already known:

$$
u^{n-1}(\mathbf{x}, t, k)=\sum_{i=1}^{n-1} X_{i}(\mathbf{x}) \cdot T_{i}(t) \cdot K_{i}(k)
$$

We wish to compute the next functional product $X_{n}(\mathbf{x}) \cdot T_{n}(t) \cdot K_{n}(k)$, which we write as $R(\mathbf{x}) \cdot S(t) \cdot W(k)$ for notational simplicity.

Thus, the solution at enrichment step $n$ reads

$$
u^{n}=u^{n-1}+R(\mathbf{x}) \cdot S(t) \cdot W(k)
$$

We propose the simplest choice for the test functions $u^{*}$ used in Eq. (9):

$$
u^{*}=R^{*}(\mathbf{x}) \cdot S(t) \cdot W(k)+R(\mathbf{x}) \cdot S^{*}(t) \cdot W(k)+R(\mathbf{x}) \cdot S(t) \cdot W^{*}(k)
$$

With the trial and test functions given by Eqs. (12) and (13) respectively, Eq. (9) is a non-linear problem that must be solved by means of a suitable iterative scheme. In our earlier papers [8] and [10], we used Newton's method. Simpler linearization strategies can also be applied, however. The simplest one is an alternated directions fixed-point algorithm, which was found remarkably robust in the present context. Each iteration consists of three steps that are repeated until reaching convergence, that is, until reaching the fixed point. The first step assumes $S(t)$ and $W(k)$ known from the previous iteration and compute an update for $R(\mathbf{x})$ (in this case the test function reduces to $\left.R^{*}(\mathbf{x}) \cdot S(t) \cdot W(k)\right)$.

In order to perform this step, the weak form is integrated in $\mathcal{I}_{t} \times \mathcal{I}_{k}$ because all the functions involving the time $t$ and the conductivity $k$ are at the present step assumed known. When the integral involves a separated form the integration is very cheap. To understand it we consider the integral of a generic function:

$$
\int_{\mathcal{I}_{t} \times \mathcal{I}_{k}} F(\mathbf{x}, t, k) d t \cdot d k
$$


For computing the integral numerically one should compute an integral in $\mathcal{I}_{t} \times \mathcal{I}_{k}$ for each value of $\mathbf{x}$. Even when considering a discrete number of points $\mathbf{x}_{k}$, the integration complexity scales with the number of points $\mathbf{x}_{k}$.

On the contrary, when the integral concerns a function that can be expressed in a separated form, the integral reduces to:

$$
\begin{gathered}
\int_{\mathcal{I}_{t} \times \mathcal{I}_{k}} F(\mathbf{x}, t, k) d t \cdot d k=\int_{\mathcal{I}_{t} \times \mathcal{I}_{k}} \sum_{j=1}^{M} F_{j}^{x}(\mathbf{x}) \cdot F_{j}^{t}(t) \cdot F_{j}^{k}(k) d t \cdot d k= \\
=\sum_{j=1}^{M} F_{j}^{x}(\mathbf{x}) \cdot\left(\int_{\mathcal{I}_{t}} F_{j}^{t}(t) d t\right) \cdot\left(\int_{\mathcal{I}_{k}} F_{j}^{k}(k) d k\right)
\end{gathered}
$$

that implies $2 \cdot M$ one-dimensional integrals.

Now, from the just-updated $R(\mathbf{x})$ and the previously-used $W(k)$, we can update $S(t)$ (with $u^{*}=R(\mathbf{x}) \cdot S^{*}(t) \cdot W(k)$ ). Finally, from the just-computed $R(\mathbf{x})$ and $S(t)$, we update $W(k)$ (with $u^{*}=R(\mathbf{x}) \cdot S(t) \cdot W^{*}(k)$ ). Again, the separability of the functions to be integrated becomes a key point from the computational point of view.

This iterative procedure continues until reaching convergence. The converged functions $R(\mathbf{x}), S(t)$ and $W(k)$ yield the new functional product at the current enrichment step: $X_{n}(\mathbf{x})=R(\mathbf{x}), T_{n}(t)=S(t)$ and $K_{n}(k)=W(k)$. The explicit form of these operations was described in many of our former works [26]. For the sake of completeness they are given in the annex.

There are other constructors of the separated representation more efficient when applying to non-symmetric differential operators, as the one based on the residual minimization, among many others [27]. The issue related to the optimality of the separated representations obtained by applying standard strategies will be addressed in the next section. The enrichment is stopped as soon as the equation residual or any other error estimator based on a quantity of interest [14] [46] is small enough.

We have seen that at each enrichment step the construction of the new functional product in Eq. (10) requires non-linear iterations. If $m_{i}$ denotes the number of iterations needed at enrichment step $i$, the total number of iterations involved in the construction of the PGD approximation is $m=\sum_{i=1}^{i=N} m_{i}$. In the above example, the entire procedure thus involves the solution of $m$ three-dimensional problems for the functions $X_{i}(\mathbf{x}), m$ one-dimensional problems for the functions $T_{i}(t)$ and $m$ algebraic systems for the functions $K_{i}(k)$. We can conclude that the complexity of the PGD procedure to compute the approximation (10) is of some tens of 3D steady-state problems (the cost related to the $1 \mathrm{D}$ and algebraic problems being negligible with respect to the 3D problems). In a classical approach, one must solve for each particular value of the parameter $k$ a 3D problem at each time step. In usual applications, this often implies the computation of several millions of 3D solutions. Clearly, the CPU time savings by applying the PGD can be of several orders of magnitude.

\subsection{Boundary conditions as extra-coordinates}

For the sake of simplicity we first consider the steady state heat equation

$$
\nabla \cdot(\mathbf{K} \cdot \nabla u(\mathbf{x}))+f(\mathbf{x})=0
$$


with $\mathbf{x} \in \Omega \subset \mathcal{R}^{3}$, subjected to the boundary conditions:

$$
\left\{\begin{array}{l}
u\left(\mathbf{x} \in \Gamma_{d}\right)=u_{g} \\
\left.(-\mathbf{K} \cdot \nabla u)\right|_{\mathbf{x} \in \Gamma_{n}} \cdot \mathbf{n}=\mathbf{q}_{g} \cdot \mathbf{n}=q_{g}
\end{array}\right.
$$

with $\mathbf{K}$ the conductivity tensor and $\mathbf{n}$ the outwards unit vector defined in the domain boundary $\Gamma_{n}$, with $\partial \Omega \equiv \Gamma=\Gamma_{d} \cup \Gamma_{n}$ and $\Gamma_{d} \cap \Gamma_{n}=\emptyset$.

\subsubsection{Neumann boundary condition as extra-coordinate}

First, imagine that we are interested in knowing the model solution for $\mathcal{M}_{q}$ values of the heat flux $q_{g}^{1}, \cdots, q_{g}^{\mathcal{M}_{q}}$ prescribed on the domain boundary $\Gamma_{n}$, i.e. $u\left(\mathbf{x} ; q_{g}^{i}\right)$, $i=1, \cdots, \mathcal{M}_{q}$. The space approximation is assumed given by a standard finite element interpolation defined from $\mathcal{M}_{x}$ nodes. As discussed previously one possibility lies in the solution of the thermal model for the different values of the prescribed flux, from which one could define a metamodel. Thus, we must calculate the solution of $\mathcal{M}_{q} 3 \mathrm{D}$ steady state heat problems and then store these solutions that involve $\mathcal{M}_{x} \cdot \mathcal{M}_{q}$ data.

Another possibility consists of considering the prescribed heat flux $q_{g}$ as an extracoordinate defined in the interval $\mathcal{I}_{q}=\left[q_{g}^{-}, q_{g}^{+}\right]$(with eventually $q_{g}^{-}=q_{g}^{1}$ and $q_{g}^{+}=$ $q_{g}^{\mathcal{M}_{q}}$ ) and then solving only once the resulting $4 \mathrm{D}$ heat equation for calculating the general parametric solution $u(\mathbf{x}, q)$. For this purpose the solution is sought in the separated form

$$
u\left(\mathbf{x}, q_{g}\right) \approx \sum_{i=1}^{N} X_{i}(\mathbf{x}) \cdot Q_{i}\left(q_{g}\right)
$$

In order to enforce the prescribed Dirichlet boundary condition $u\left(\mathbf{x} \in \Gamma_{d}\right)=u_{g}$ the simplest procedure consists of choosing the first functional couple $X_{1}(\mathbf{x}) \cdot Q_{1}\left(q_{g}\right)$ in order to ensure that $u^{1}\left(\mathbf{x} \in \Gamma_{d}, q_{g}\right)=X_{1}\left(\mathbf{x} \in \Gamma_{d}\right) \cdot Q_{1}\left(q_{g}\right)=u_{g}$. Thus, the remaining terms of the finite sum $X_{i}(\mathbf{x}), i>1$, will be subjected to homogeneous essential boundary conditions, i.e. $X_{i}\left(\mathbf{x} \in \Gamma_{d}\right)=0, i>1$. Alternative possibilities for addressing Dirichlet boundary conditions consist of using penalty or Lagrange multiplier formulations. Other possibilities were considered in [37].

In order to use the approximation (18) we start by considering the weak form related to Eq. (16), that writes: Find $u(\mathbf{x}) \in H^{1}(\Omega)$, verifying $u\left(\mathbf{x} \in \Gamma_{d}\right)=u_{g}$, such that

$$
\int_{\Omega} \nabla u^{*} \cdot(\mathbf{K} \cdot \nabla u) d \mathbf{x}=\int_{\Gamma_{n}} u^{*} \cdot(\mathbf{K} \cdot \nabla u) \cdot \mathbf{n} d \mathbf{x}+\int_{\Omega} u^{*} \cdot f(\mathbf{x}) d \mathbf{x}
$$

is verified $\forall u^{*} \in H^{1}(\Omega)$, with $u^{*}\left(\mathbf{x} \in \Gamma_{d}\right)=0$.

By introducing the Neumann condition given in (17) into (19) it results

$$
\int_{\Omega} \nabla u^{*} \cdot(\mathbf{K} \cdot \nabla u) d \mathbf{x}=-\int_{\Gamma_{n}} u^{*} \cdot q_{g} d \mathbf{x}+\int_{\Omega} u^{*} \cdot f(\mathbf{x}) d \mathbf{x}
$$

For using the approximation (18) we must consider the extended-weak form defined in the domain $\Omega \times \mathcal{I}_{q}$

$$
\int_{\Omega \times \mathcal{I}_{q}} \nabla u^{*} \cdot(\mathbf{K} \cdot \nabla u) d \mathbf{x} \cdot d q_{g}=-\int_{\Gamma_{n} \times \mathcal{I}_{q}} u^{*} \cdot q_{g} d \mathbf{x} \cdot d q_{g}+\int_{\Omega \times \mathcal{I}_{q}} u^{*} \cdot f(\mathbf{x}) d \mathbf{x} \cdot d q_{g}
$$


that assuming at iteration $n$ :

$$
\left\{\begin{aligned}
u^{n}\left(\mathbf{x}, q_{g}\right)= & \sum_{i=1}^{n-1} X_{i}(\mathbf{x}) \cdot Q_{i}\left(q_{g}\right)+X_{n}(\mathbf{x}) \cdot Q_{n}\left(q_{g}\right)= \\
& =u^{n-1}\left(\mathbf{x}, q_{g}\right)+X_{n}(\mathbf{x}) \cdot Q_{n}\left(q_{g}\right) \\
u^{*}= & X^{*}(\mathbf{x}) \cdot Q_{n}\left(q_{g}\right)+X_{n}(\mathbf{x}) \cdot Q^{*}\left(q_{g}\right)
\end{aligned}\right.
$$

with $u^{n-1}\left(\mathbf{x}, q_{g}\right)$ known, we can compute the new couple of unknown functions $X_{n}(\mathbf{x})$ and $Q_{n}\left(q_{g}\right)$ by applying the alternated directions fixed point algorithm just summarized and described in the annex for a generic parametric problem, that is assumed to reach the fixed point with a prescribed precision in $m_{n}$ iterations.

If we assume that the solution needs $N$ terms in the finite sum, the solution will involve $N \cdot\left(\mathcal{M}_{x}+\mathcal{M}_{q}\right)$ data. If we define $m=\sum_{i=1}^{i=N} m_{i}$ the separated representation solution need the solution of $m$ 3D problems for calculating functions $X_{i}(\mathbf{x}), i=$ $1, \cdots N$, and $m$ 1D problems for calculating the functions $Q_{i}\left(q_{g}\right), i=1, \cdots, N$. The computing cost related to the solution of the $1 \mathrm{D}$ problems can be neglected with respect to the one associated with the solution of the 3D problems. Thus, if $m<\mathcal{M}_{q}$, PGD will proceed faster than the solution of the model for the different values of the parameter $q_{g}^{i}, i=1, \cdots, \mathcal{M}_{q}$. From the point of view of the data storage, PGD is superior as soon as $N \cdot\left(\mathcal{M}_{x}+\mathcal{M}_{q}\right)<\mathcal{M}_{x} \cdot \mathcal{M}_{q}$.

When considering only one parameter as extra-coordinate the superiority of PGD with respect to standard procedures is not crucial, but as discussed previously, when the number of extra-coordinates increases the benefit in using the PGD is impresive.

\subsubsection{Dirichlet boundary condition as extra-coordinate}

In this section we consider that we are interested in considering the solution of model (16) for any value of $u_{g}$ in (17) in a certain interval $\mathcal{I}_{u}=\left[u_{g}^{-}, u_{g}^{+}\right]$. For this purpose we consider the function $\varphi(\mathbf{x})$ continuous in $\bar{\Omega}$ such that $\Delta \varphi \in L_{2}(\Omega)$ and $\varphi\left(\mathbf{x} \in \Gamma_{d}\right)=1$. Thus, we can define the change of variable [37]

$$
u(\mathbf{x})=v(\mathbf{x})+u_{g} \cdot \varphi(\mathbf{x})
$$

that allows rewriting Eqs. (16) and (17) as:

$$
\nabla \cdot(\mathbf{K} \cdot \nabla v(\mathbf{x}))+u_{g} \cdot \nabla \cdot(\mathbf{K} \cdot \nabla \varphi(\mathbf{x}))+f(\mathbf{x})=0
$$

subjected to the boundary conditions:

$$
\left\{\begin{array}{l}
v\left(\mathbf{x} \in \Gamma_{d}\right)=0 \\
\left.(-\mathbf{K} \cdot \nabla v)\right|_{\mathbf{x} \in \Gamma_{n}} \cdot \mathbf{n}=\left.u_{g} \cdot(\mathbf{K} \cdot \nabla \varphi)\right|_{\mathbf{x} \in \Gamma_{n}} \cdot \mathbf{n}+q_{g}
\end{array}\right.
$$

that results in the weak form

$$
\begin{gathered}
\int_{\Omega} \nabla u^{*} \cdot(\mathbf{K} \cdot \nabla v) d \mathbf{x}=-\int_{\Omega} \nabla u^{*} \cdot u_{g} \cdot(\mathbf{K} \cdot \nabla \varphi) d \mathbf{x}+\int_{\Omega} u^{*} \cdot f(\mathbf{x}) d \mathbf{x}- \\
-\int_{\Gamma_{n}} u^{*} \cdot q_{g} d \mathbf{x}-\int_{\Gamma_{n}} u^{*} \cdot u_{g} \cdot(\mathbf{K} \cdot \nabla \varphi) \cdot \mathbf{n} d \mathbf{x}
\end{gathered}
$$

that in fact only requires $\mathcal{C}^{0}$ continuity of the function $\varphi(\mathbf{x})$. 
We can now introduce $u_{g}$ as extra-coordinate, searching the solution in the separated form:

$$
u\left(\mathbf{x}, u_{g}\right) \approx \sum_{i=1}^{N} X_{i}(\mathbf{x}) \cdot U_{i}\left(u_{g}\right)
$$

that needs for the extended weak-form

$$
\begin{gathered}
\int_{\Omega \times \mathcal{I}_{u}} \nabla u^{*} \cdot(\mathbf{K} \cdot \nabla v) d \mathbf{x} \cdot d u_{g}= \\
=-\int_{\Omega \times \mathcal{I}_{u}} \nabla u^{*} \cdot u_{g} \cdot(\mathbf{K} \cdot \nabla \varphi) d \mathbf{x} \cdot d u_{g}+\int_{\Omega \times \mathcal{I}_{u}} u^{*} \cdot f(\mathbf{x}) d \mathbf{x} \cdot d u_{g}- \\
-\int_{\Gamma_{n} \times \mathcal{I}_{u}} u^{*} \cdot q_{g} d \mathbf{x} \cdot d u_{g}-\int_{\Gamma_{n} \times \mathcal{I}_{u}} u^{*} \cdot u_{g} \cdot(\mathbf{K} \cdot \nabla \varphi) \cdot \mathbf{n} d \mathbf{x} \cdot d u_{g}
\end{gathered}
$$

on which the alternated directions fixed point algorithm applies again to calculate the parametric solution (27).

\subsubsection{Mixed boundary conditions as extra-coordinates}

From the extended weak form

$$
\begin{gathered}
\int_{\Omega \times \mathcal{I}_{u} \times \mathcal{I}_{q}} \nabla u^{*} \cdot(\mathbf{K} \cdot \nabla v) d \mathbf{x} \cdot d u_{g} \cdot d q_{g}= \\
=-\int_{\Omega \times \mathcal{I}_{u} \times \mathcal{I}_{q}} \nabla u^{*} \cdot u_{g} \cdot(\mathbf{K} \cdot \nabla \varphi) d \mathbf{x} \cdot d u_{g} \cdot d q_{g}+\int_{\Omega \times \mathcal{I}_{u} \times \mathcal{I}_{q}} u^{*} \cdot f(\mathbf{x}) d \mathbf{x} \cdot d u_{g} \cdot d q_{g}- \\
-\int_{\Gamma_{n} \times \mathcal{I}_{u} \times \mathcal{I}_{q}} u^{*} \cdot q_{g} d \mathbf{x} \cdot d u_{g} \cdot d q_{g}-\int_{\Gamma_{n} \times \mathcal{I}_{u} \times \mathcal{I}_{q}} u^{*} \cdot u_{g} \cdot(\mathbf{K} \cdot \nabla \varphi) \cdot \mathbf{n} d \mathbf{x} \cdot d u_{g} \cdot d q_{g}(29)
\end{gathered}
$$

one could compute a parametric solution involving Dirichlet and Neumann boundary conditions as extra-coordinates, i.e. $u\left(\mathbf{x}, u_{g}, q_{g}\right)$ according to:

$$
u\left(\mathbf{x}, u_{g}, q_{g}\right) \approx \sum_{i=1}^{N} X_{i}(\mathbf{x}) \cdot U_{i}\left(u_{g}\right) \cdot Q_{i}\left(q_{g}\right)
$$

\subsubsection{Non constant Neumann boundary conditions}

We consider that in Eq. (17) $q_{g}=q_{g}(\mathbf{x})$, with $\mathbf{x} \in \Gamma_{n}$. First we assume that $q_{g}(\mathbf{x})$ can be approximated on $\Gamma_{n}$ from:

$$
q_{g}(\mathbf{x}) \approx \sum_{i=1}^{S_{q}} Q_{g}^{k} \cdot \xi_{k}(\mathbf{x})
$$

where $\mathbf{x} \in \Gamma_{n}$ and $Q_{g}^{k}$ represents the prescribed nodal fluxes, i.e. $Q_{g}^{k}=g_{g}\left(\mathbf{x}_{k}\right)$ at the nodal positions $\mathbf{x}_{k} \in \Gamma_{n}$.

Now, introducing the approximation (31) into the weak form (20) it results

$$
\int_{\Omega} \nabla u^{*} \cdot(\mathbf{K} \cdot \nabla u) d \mathbf{x}=-\int_{\Gamma_{n}} u^{*} \cdot\left(\sum_{i=1}^{i=S_{q}} Q_{g}^{k} \cdot \xi_{k}(\mathbf{x})\right) d \mathbf{x}+\int_{\Omega} u^{*} \cdot f(\mathbf{x}) d \mathbf{x}
$$


If the nodal fluxes $Q_{g}^{k}, k=1, \cdots, S_{q}$, can take values into the intervals $\mathcal{I}_{Q}^{k}$ and we are interested to calculating the general parametric solution $u\left(\mathbf{x}, Q_{g}^{1}, \cdots, Q_{g}^{S_{q}}\right)$, it suffices to consider the extended weak form related to Eq. (32)

$$
\begin{gathered}
\int_{\Omega \times \mathcal{I}_{Q}^{1} \times \cdots \times \mathcal{I}_{Q}^{S_{q}}} \nabla u^{*} \cdot(\mathbf{K} \cdot \nabla u) d \mathbf{x} \cdot d Q_{g}^{1} \cdots d Q_{g}^{S_{q}}= \\
=-\int_{\Gamma_{n} \times \mathcal{I}_{Q}^{1} \times \cdots \times \mathcal{I}_{Q}^{S_{q}}} u^{*} \cdot\left(\sum_{i=1}^{S_{q}} Q_{g}^{k} \cdot \xi_{k}(\mathbf{x})\right) d \mathbf{x} \cdot d Q_{g}^{1} \cdots d Q_{g}^{S_{q}}+ \\
+\int_{\Omega \times \mathcal{I}_{Q}^{1} \times \cdots \times \mathcal{I}_{Q}^{S_{q}}} u^{*} \cdot f(\mathbf{x}) d \mathbf{x} \cdot d Q_{g}^{1} \cdots d Q_{g}^{S_{q}}
\end{gathered}
$$

and the solution separated representation

$$
u\left(\mathbf{x}, Q_{g}^{1}, \cdots, Q_{g}^{S_{q}}\right) \approx \sum_{i=1}^{N} X_{i}(\mathbf{x}) \cdot \prod_{j=1}^{S_{q}} G_{i}^{j}\left(Q_{g}^{j}\right)
$$

Then, from Eqs. (33) and (34) we can compute the parametric solution. In this case the use of the separated representation is compulsory because the curse of dimensionality that model (33) implies when $S_{q}$ increases. Standard discretization strategies fail for solving the multi-dimensional model (33) and the sampling of the parametric space becomes inefficient when its dimensionality increases.

\subsubsection{Non constant Dirichlet boundary consitions}

In this section we consider the solution of model (16) for $u_{g}$ in Eq. (17) depending on the space, i.e. $u_{g}(\mathbf{x}), \mathbf{x} \in \Gamma_{d}$. If $u_{g}(\mathbf{x})$ can be approximated on $\Gamma_{d}$ from

$$
u_{g}(\mathbf{x}) \approx \sum_{k=1}^{S_{u}} U_{g}^{k} \cdot \eta_{k}(\mathbf{x})
$$

where $U_{g}^{k}$ represents the prescribed nodal temperatures, i.e. $U_{g}^{k}=u_{g}\left(\mathbf{x}_{k}\right)$ at the nodal positions $\mathbf{x}_{k} \in \Gamma_{d}$ taking values in the intervals $\mathcal{I}_{U}^{k}$.

To compute the parametric solution $u\left(\mathbf{x}, U_{g}^{1}, \cdots, U_{g}^{S_{u}}\right)$ we first define the functions $\varphi_{k}(\mathbf{x})$ continuous in $\bar{\Omega}$ and verifying $\Delta \varphi(\mathbf{x}) \in L_{2}(\Omega)$, such that $\varphi_{k}\left(\mathbf{x} \in \Gamma_{d}\right)=\eta_{k}(\mathbf{x})$. Thus, we can define the change of variable

$$
u(\mathbf{x})=v(\mathbf{x})+\sum_{i=1}^{S_{u}} U_{g}^{k} \cdot \varphi_{k}(\mathbf{x})
$$

that leads to the weak form

$$
\begin{aligned}
\int_{\Omega} \nabla v^{*} \cdot(\mathbf{K} & \cdot \nabla v) d \mathbf{x}=-\int_{\Omega} \nabla v^{*} \cdot\left(\sum_{k=1}^{S_{u}} U_{g}^{k} \cdot\left(\mathbf{K} \cdot \nabla \varphi_{k}\right)\right) d \mathbf{x}+\int_{\Omega} v^{*} \cdot f(\mathbf{x}) d \mathbf{x}- \\
& -\int_{\Gamma_{n}} v^{*} \cdot q_{g} d \mathbf{x}-\int_{\Gamma_{n}} v^{*} \cdot\left(\sum_{k=1}^{S_{u}} U_{g}^{k} \cdot\left(\mathbf{K} \cdot \nabla \varphi_{k}\right) \cdot \mathbf{n}\right) d \mathbf{x}
\end{aligned}
$$


We can consider now the separated form of the parametric solution

$$
u\left(\mathbf{x}, U_{g}^{1}, \cdots, U_{g}^{S_{u}}\right) \approx \sum_{i=1}^{N} X_{i}(\mathbf{x}) \cdot \prod_{j=1}^{S_{u}} F_{i}^{j}\left(U_{g}^{j}\right)
$$

that will be constructed using some standard procedure (alternated directions fixed point or residual minimization) from the extended weak form:

$$
\begin{gathered}
\int_{\Omega \times \mathcal{I}_{U}^{1} \times \cdots \times \mathcal{I}_{U}^{S_{u}}} \nabla u^{*} \cdot(\mathbf{K} \cdot \nabla v) d \mathbf{x} \cdot d U_{g}^{1} \cdots d U_{g}^{S_{u}}= \\
=-\int_{\Omega \times \mathcal{I}_{U}^{1} \times \cdots \times \mathcal{I}_{U}^{S_{u}}} \nabla u^{*} \cdot\left(\sum_{i=1}^{S_{u}} U_{g}^{k} \cdot\left(\mathbf{K} \cdot \nabla \varphi_{k}\right)\right) d \mathbf{x} \cdot d U_{g}^{1} \cdots d U_{g}^{S_{u}+} \\
+\int_{\Omega \times \mathcal{I}_{U}^{1} \times \cdots \times \mathcal{I}_{U}^{S_{u}}} u^{*} \cdot f(\mathbf{x}) d \mathbf{x} \cdot d U_{g}^{1} \cdots d U_{g}^{S_{u}}-\int_{\Gamma_{n} \times \mathcal{I}_{U}^{1} \times \cdots \times \mathcal{I}_{U}^{S_{u}}} u^{*} \cdot q_{g} d \mathbf{x} \cdot d U_{g}^{1} \cdots d U_{g}^{S_{u}} \\
-\int_{\Gamma_{n} \times \mathcal{I}_{U}^{1} \times \cdots \times \mathcal{I}_{U}^{S u}} u^{*} \cdot\left(\sum_{i=1}^{S_{u}} U_{g}^{k} \cdot\left(\mathbf{K} \cdot \nabla \varphi_{k}\right) \cdot \mathbf{n}\right) d \mathbf{x} \cdot d U_{g}^{1} \cdots d U_{g}^{S_{u}}
\end{gathered}
$$

\subsection{Initial conditions as extra-coordinates}

We consider in this section the transient heat equation in a homogeneous and isotropic medium

$$
\rho \cdot C_{p} \cdot \frac{\partial u}{\partial t}=k \cdot \Delta u+f
$$

$t \in \mathcal{I}_{t}=(0, \Theta] \subset \mathcal{R}, \mathbf{x} \in \Omega \subset \mathcal{R}^{3}$ and $f=$ cte. The initial and boundary conditions read:

$$
\left\{\begin{array}{l}
u\left(\mathbf{x} \in \Gamma_{d}\right)=u_{g} \\
\left.(-k \cdot \nabla u)\right|_{\mathbf{x} \in \Gamma_{n}} \cdot \mathbf{n}=q_{g} \\
u(\mathbf{x}, t=0)=u^{0}(\mathbf{x})
\end{array}\right.
$$

The associated weak form reads:

$$
\int_{\Omega} u^{*} \cdot \rho \cdot C_{p} \cdot \frac{\partial u}{\partial t} d \mathbf{x}+\int_{\Omega} \nabla u^{*} \cdot k \cdot \nabla u d \mathbf{x}=-\int_{\Gamma_{n}} u^{*} \cdot q_{g} d \mathbf{x}+\int_{\Omega} u^{*} \cdot f(\mathbf{x}) d \mathbf{x}
$$

that includes explicitly the natural (Neumann) boundary conditions. To prescribe both the initial and the essential (Dirichlet) boundary conditions we proceed to define the following functions:

$$
\begin{gathered}
\hat{u}^{0}(\mathbf{x})= \begin{cases}u^{0}(\mathbf{x}), & \mathbf{x} \in \Omega \\
0, & \mathbf{x} \in \Gamma\end{cases} \\
\Upsilon(t)=\left\{\begin{array}{l}
1, t>0 \\
0, t=0
\end{array}\right.
\end{gathered}
$$

and $\varphi(\mathbf{x})$ continuous in $\bar{\Omega}$, verifying $\Delta \varphi \in L_{2}(\Omega)$ and the essential boundary conditions

$$
\varphi\left(\mathbf{x} \in \Gamma_{d}\right)=u_{g}
$$


We could define the function $\Sigma(\mathbf{x}, t)$ expressed in the separated form

$$
\Sigma(\mathbf{x}, t)=\hat{u}^{0}(\mathbf{x})+\varphi(\mathbf{x}) \cdot \Upsilon(t)
$$

that verifies the initial and essential boundary conditions. However, functions $\hat{u}^{0}$ and $\Upsilon(t)$ are not regular enough to be employed in the weak form of the problem. A direct regularization consists in defining these functions at the nodal positions and then define interpolations with the required regularity. Thus, the discrete counterpart of functions $\hat{u}^{0}$ and $\Upsilon(t)$ are given by:

$$
\hat{u}^{0}\left(\mathbf{x}_{k}\right)= \begin{cases}u^{0}\left(\mathbf{x}_{k}\right), & \mathbf{x}_{k} \in \Omega \\ 0, & \mathbf{x}_{k} \in \Gamma\end{cases}
$$

and

$$
\Upsilon\left(t_{l}\right)=\left\{\begin{array}{l}
1, t_{l}>0 \\
0, t_{l}=0
\end{array}\right.
$$

with $k=1, \cdots 1, \cdots, \mathcal{M}_{x} ; l=1, \cdots, \mathcal{M}_{t}$. Now, standard interpolation is applied to the define functions $\hat{u}^{0}(\mathbf{x})$ and $\Upsilon(t)$ everywhere from theirs nodal values expressed by Eqs. (47) and (48).

By applying now the change of variable:

$$
u(\mathbf{x}, t)=v(\mathbf{x}, t)+\Sigma(\mathbf{x}, t)=v(\mathbf{x}, t)+\hat{u}^{0}(\mathbf{x})+\varphi(\mathbf{x}) \cdot \Upsilon(t)
$$

the weak form (57) results:

$$
\begin{aligned}
\int_{\Omega} v^{*} \cdot \rho \cdot C_{p} \cdot \frac{\partial v}{\partial t} d \mathbf{x}+\int_{\Omega} \nabla v^{*} \cdot k \cdot \nabla v d \mathbf{x}= \\
=-\int_{\Omega} v^{*} \cdot \rho \cdot C_{p} \cdot \varphi \cdot \frac{\partial \Upsilon}{\partial t} d \mathbf{x}- \\
\quad-\int_{\Omega} \nabla v^{*} \cdot k \cdot \nabla \hat{u}^{0} d \mathbf{x}- \\
\quad-\int_{\Gamma_{n}} v^{*} \cdot k \cdot \nabla \hat{u}^{0} \cdot \mathbf{n} d \mathbf{x}- \\
-\int_{\Gamma_{n}} v^{*} \cdot q_{g} d \mathbf{x}-\int_{\Omega} \nabla v^{*} \cdot k \cdot \Upsilon \cdot \nabla \varphi d \mathbf{x}- \\
-\int_{\Gamma_{n}} v^{*} \cdot k \cdot \Upsilon \cdot \nabla \varphi \cdot \mathbf{n} d \mathbf{x}+\int_{\Omega} v^{*} \cdot f(\mathbf{x}) d \mathbf{x}
\end{aligned}
$$

If the initial condition is approximated by

$$
\hat{u}^{0}(\mathbf{x}) \approx \sum_{k=1}^{S_{0}} U_{0}^{k} \cdot \eta_{k}(\mathbf{x})
$$

after introducing it into the weak form (50) we obtain:

$$
\begin{gathered}
\int_{\Omega} v^{*} \cdot \rho \cdot C_{p} \cdot \frac{\partial v}{\partial t} d \mathbf{x}+\int_{\Omega} \nabla v^{*} \cdot k \cdot \nabla v d \mathbf{x}= \\
=-\int_{\Omega} v^{*} \cdot \rho \cdot C_{p} \cdot \varphi \cdot \frac{\partial \Upsilon}{\partial t} d \mathbf{x}-
\end{gathered}
$$




$$
\begin{gathered}
-\int_{\Omega} \nabla v^{*} \cdot k \cdot\left(\sum_{k=1}^{S_{0}} U_{0}^{k} \cdot \nabla \eta_{k}(\mathbf{x})\right) d \mathbf{x}- \\
-\int_{\Gamma_{n}} v^{*} \cdot k \cdot\left(\sum_{k=1}^{S_{0}} U_{0}^{k} \cdot \eta_{k}(\mathbf{x}) \cdot \mathbf{n}\right) d \mathbf{x}- \\
-\int_{\Gamma_{n}} v^{*} \cdot q_{g} d \mathbf{x}-\int_{\Omega} \nabla v^{*} \cdot k \cdot \Upsilon \cdot \nabla \varphi d \mathbf{x}- \\
-\int_{\Gamma_{n}} v^{*} \cdot k \cdot \Upsilon \cdot \nabla \varphi \cdot \mathbf{n} d \mathbf{x}+\int_{\Omega} v^{*} \cdot f(\mathbf{x}) d \mathbf{x}
\end{gathered}
$$

that allows us looking for a parametric solution $v\left(\mathbf{x}, U_{0}^{1}, \cdots, U_{0}^{S_{0}}\right)$, with $U_{0}^{j} \in \mathcal{I}_{0}^{j}=$ $\left[\left(U_{0}^{j}\right)^{-},\left(U_{0}^{j}\right)^{+}\right]$

$$
u\left(\mathbf{x}, U_{0}^{1}, \cdots, U_{0}^{S_{0}}\right) \approx \sum_{i=1}^{N} X_{i}(\mathbf{x}) \cdot \prod_{j=1}^{S_{0}} \mathcal{U}_{i}^{j}\left(U_{0}^{j}\right)
$$

from the extended weak form

$$
\begin{aligned}
& \int_{\Omega \times \mathcal{I}_{0}^{1} \times \cdots \times \mathcal{I}_{0}^{S_{0}}} v^{*} \cdot \rho \cdot C_{p} \cdot \frac{\partial v}{\partial t} d \mathbf{x} \cdot d U_{0}^{1} \cdots d U_{0}^{S_{0}}+ \\
& +\int_{\Omega \times \mathcal{I}_{0}^{1} \times \cdots \times \mathcal{I}_{0}^{S_{0}}} \nabla v^{*} \cdot k \cdot \nabla v d \mathbf{x} \cdot d U_{0}^{1} \cdots d U_{0}^{S_{0}}= \\
& =-\int_{\Omega \times \mathcal{I}_{0}^{1} \times \cdots \times \mathcal{I}_{0}^{S_{0}}} v^{*} \cdot \rho \cdot C_{p} \cdot \varphi \cdot \frac{\partial \Upsilon}{\partial t} d \mathbf{x} \cdot d U_{0}^{1} \cdots d U_{0}^{S_{0}}- \\
& -\int_{\Omega \times \mathcal{I}_{0}^{1} \times \cdots \times \mathcal{I}_{0}^{S_{0}}} \nabla v^{*} \cdot k \cdot\left(\sum_{k=1}^{S_{0}} U_{0}^{k} \cdot \nabla \eta_{k}(\mathbf{x})\right) d \mathbf{x} \cdot d U_{0}^{1} \cdots d U_{0}^{S_{0}}- \\
& -\int_{\Gamma_{n} \times \mathcal{I}_{0}^{1} \times \cdots \times \mathcal{I}_{0}^{S_{0}}} v^{*} \cdot k \cdot\left(\sum_{k=1}^{S_{0}} U_{0}^{k} \cdot \eta_{k}(\mathbf{x}) \cdot \mathbf{n}\right) d \mathbf{x} \cdot d U_{0}^{1} \cdots d U_{0}^{S_{0}}- \\
& -\int_{\Gamma_{n} \times \mathcal{I}_{0}^{1} \times \cdots \times \mathcal{I}_{0}^{S_{0}}} v^{*} \cdot q_{g} d \mathbf{x} \cdot d U_{0}^{1} \cdots d U_{0}^{S_{0}}- \\
& \int_{\Omega \times \mathcal{I}_{0}^{1} \times \cdots \times \mathcal{I}_{0}^{S_{0}}} \nabla v^{*} \cdot k \cdot \Upsilon \cdot \nabla \varphi d \mathbf{x} \cdot d U_{0}^{1} \cdots d U_{0}^{S_{0}}- \\
& -\int_{\Gamma_{n} \times \mathcal{I}_{0}^{1} \times \cdots \times \mathcal{I}_{0}^{S_{0}}} v^{*} \cdot k \cdot \Upsilon \cdot \nabla \varphi \cdot \mathbf{n} d \mathbf{x} \cdot d U_{0}^{1} \cdots d U_{0}^{S_{0}}+ \\
& +\int_{\Omega \times \mathcal{I}_{0}^{1} \times \cdots \times \mathcal{I}_{0}^{S_{0}}} v^{*} \cdot f(\mathbf{x}) d \mathbf{x} \cdot d U_{0}^{1} \cdots d U_{0}^{S_{0}}
\end{aligned}
$$


3.4 Geometrical parameters as extra-coordinates

For the sake of clarity and without loss of generality we are addressing in this section the transient one-dimensional heat equation

$$
\frac{\partial u}{\partial t}=\alpha \cdot \frac{\partial^{2} u}{\partial x^{2}}+f
$$

with $t \in \mathcal{I}_{t}=(0, \Theta] \subset \mathcal{R}, x \in \Omega=(0, L) \subset \mathcal{R}, f=$ cte and $u(x=0, t)=u(x=L, t)=$ $u(x, t=0)=0$.

The associated space-time weak form reads:

$$
\int_{\Omega \times \mathcal{I}_{t}} u^{*} \cdot \frac{\partial u}{\partial t} d x \cdot d t=-\alpha \cdot \int_{\Omega \times \mathcal{I}_{t}} \frac{\partial u^{*}}{\partial x} \cdot \frac{\partial u}{\partial x} d x \cdot d t+\int_{\Omega \times \mathcal{I}_{t}} u^{*} \cdot f d x \cdot d t
$$

If we are interested in computing the solution $u(x, t)$ in many domains of length $L \in\left[L^{-}, L^{+}\right]$and for many time intervals of length $\Theta=\left[\Theta^{-}, \Theta^{+}\right]$, more than solving the model for many possible choices in order to define a metamodel, it is preferable to compute the parametric solution $u(x, t, L, \Theta)$.

This parametric solution is sought in the separated form

$$
u(x, t, L, \Theta) \approx \sum_{i=1}^{N} X_{i}(x) \cdot T_{i}(t) \cdot \mathcal{L}_{i}(L) \cdot \mathcal{T}_{i}(\Theta)
$$

However, Eq. (56) does not involve an explicit dependence on the extra-coordinates $L$ and $\Theta$, both defining the domain of integration. In order to explicit this dependence, we consider the coordinates transformation

$$
\left\{\begin{array}{l}
t=\tau \cdot \Theta, \tau \in[0,1] \\
x=\lambda \cdot L, \lambda \in[0,1]
\end{array}\right.
$$

In this case the weak form (56) reads:

$$
\int_{[0,1]^{2}} u^{*} \cdot \frac{\partial u}{\partial \tau} \cdot L d \lambda \cdot d \tau=-\alpha \cdot \int_{[0,1]^{2}} \frac{\partial u^{*}}{\partial \lambda} \cdot \frac{\partial u}{\partial \lambda} \cdot \frac{\Theta}{L} d \lambda \cdot d \tau+\int_{[0,1]^{2}} u^{*} \cdot f \cdot L \cdot \Theta d \lambda \cdot d \tau
$$

that allows calculating the parametric solution derived from (57) after applying the change of coordinates

$$
u(\lambda, \tau, L, \Theta) \approx \sum_{i=1}^{N} \tilde{X}_{i}(\lambda) \cdot \tilde{T}_{i}(\tau) \cdot \mathcal{L}_{i}(L) \cdot \mathcal{T}_{i}(\Theta)
$$

\section{Virtual charts for industrial applications}

As just illustrated usual computational mechanics models could be enriched by introducing several extra-coordinates. Thus, adding some new coordinates to models initially non high-dimensional, could lead to new, never before explored insights in the physics as previously illustrated in the context of a parametric thermal models.

Next, we review some of the most representative examples explored so far. 


\subsection{Geometrical and material parameters}

Classical design strategies consider given parameters and then solve the mechanical problem. A cost function is evaluated as soon as the solution is available. If the solution is not good enough, parameters are updated by using an appropriate optimization strategy and then the model is solved again, and the process continues until reaching converence. The main drawback lies in the fact that numerous resolutions are generally needed with the consequent impact in terms of the computing time.

As explained before, if all the parameters involved in the design process are considered as extra-coordinates (just like space and time in standard models) a unique solution of the resulting multidimensional model allows knowing the solution for any choice of the parameters considered as extra-coordinates. The price to pay is the solution of a multidimensional model. However, this solution is feasible by invoking the PGD solver and its inherent separated representation. This allows circumventing the curse of dimensionality.

This kind of parametric modelling was addressed in [15] [66] [17] [28] where material parameters were introduced as extra-coordinates. In [47], thermal conductivities, macroscopic temperature and its time evolution were introduced as extra-coordinates for computing linear and non-linear homogenization.

In [21] we proved that the PGD method with separated space coordinates is a very efficient way to compute 3D elastic problems defined in degenerated domains (plate or shells) with a numerical cost that scales like 2D. The key point for such an approach is to use a separated representation for each quantity of the model as a sum of products of functions of each coordinate or group of coordinates. In he case of a plate the retained separated representation of a generic function $u(x, y, z)$ reads:

$$
u(x, y, z) \approx \sum_{i=1}^{i=N} X_{i}(x, y) \cdot Z_{i}(z)
$$

In this work, we consider additional model parameters as extra-coordnates. In addition to the 3 dimensions describing the physical space, we add new coordinates related to the Young's modulus $E$, to the Poisson's coefficient $\nu$ and to the geometrical parameter $e$ depicted in Fig. 1. Thus separated representations write:

$$
u(x, y, z, E, \nu, e) \approx \sum_{i=1}^{i=N} X_{i}(x, y) \cdot Z_{i}(z) \cdot F_{i}(E) \cdot H_{i}(\nu) \cdot G_{i}(e)
$$

For and efficient solution of the mechanical model making use of a separated representation we must ensure a separated representation of all the fields involved in the model. However, there is a technical difficulty because the coordinates $e$ and $z$ are not independent. In order to perform a fully separated representation we could consider the following transformation $z \rightarrow z^{\prime}$ :

$$
\begin{cases}z^{\prime}=\frac{z}{e} & z \in[0, e] \\ z^{\prime}=1+\frac{z-e}{h} & z \in[e, e+h] \\ z^{\prime}=2+\frac{z-h-e}{e} & z \in[e+h, e+h+e]\end{cases}
$$

Thus, finally $z^{\prime} \in[0,3]$ and $e \in \Omega_{e}$, both being independents, lead to a fully separated representation. The components of the Jacobian matrix are $\frac{1}{e}$ or $\frac{1}{h}$ that facilitates the change of variable in the resulting weak form related to the elastic model. 


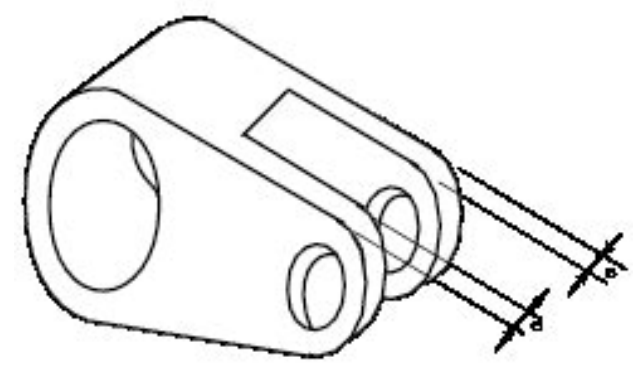

Fig. 1 Parametrized part.
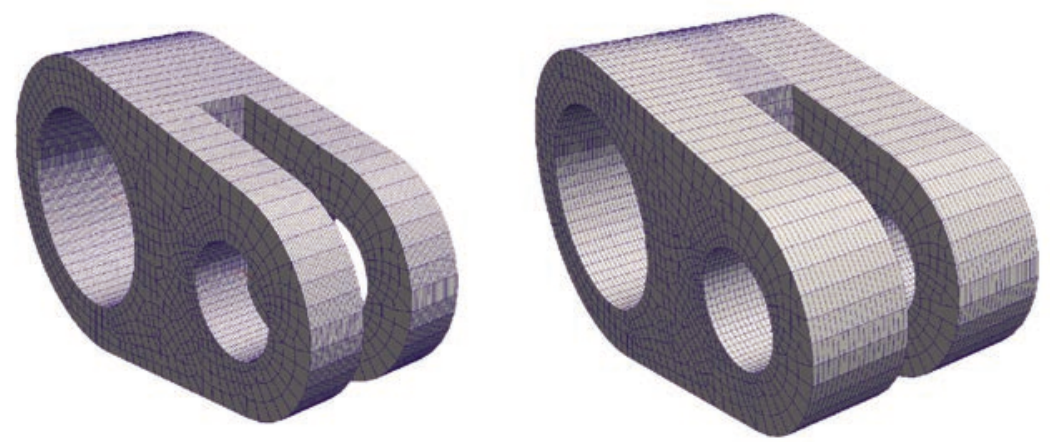

Fig. 2 Parts related to different choices of the model parameter $e$.

In the numerical example here addressed we considered $\nu \in[0,0.5], E \in[5,500](G P a)$ and $e \in[5,20](\mathrm{mm})$ that allow to describe a large variety of isotropic material: plastics, metals, alloys, ...

As soon the parametric solution is computed by solving only once the resulting multidimensional model (defined in this case in a space of dimension 6) we can particularize it for different materials (by choosing appropriate values of $E$ or $\nu$ ) or for different geometries (by choosing $e$ ). Fig. 2 illustrates the same part for two values of the parameter $e$.

In [21], the anisotropy directions of plies involved in a composite laminate were considered as extra-coordinates. As soon as the separated representation of the parametric solution was computed off-line, its on-line use only needs to particularize such solution for a desired set of parameters. Obviously, this task can be performed very fast, many times in real time, and by using light computing platforms, as smartphones or tablets. Fig. 3 illustrates a smartphone application [21] in which the elastic solution of a two-plies composite laminate was computed by introducing the orientation of fiber in each ply, $\theta_{1}$ and $\theta_{2}$, as extra-coordinates

$$
u_{j}\left(x, y, z, \theta_{1}, \theta_{2}\right) \approx \sum_{i=1}^{N} X_{i}^{j}(x, y) \cdot Z_{i}^{j}(z) \cdot \Theta_{i}^{j, 1}\left(\theta_{1}\right) \cdot \Theta_{i}^{j, 2}\left(\theta_{2}\right)
$$

Then one can visualize each component of the displacement field, by particularizing the $z$-coordinate from the horizontal slider as well as the orientation of the reinforcement in both plies from both vertical sliders. Obviously when the laminate is equilibrated there is no noticeable deformations and the plate remains plane, but as soon as 


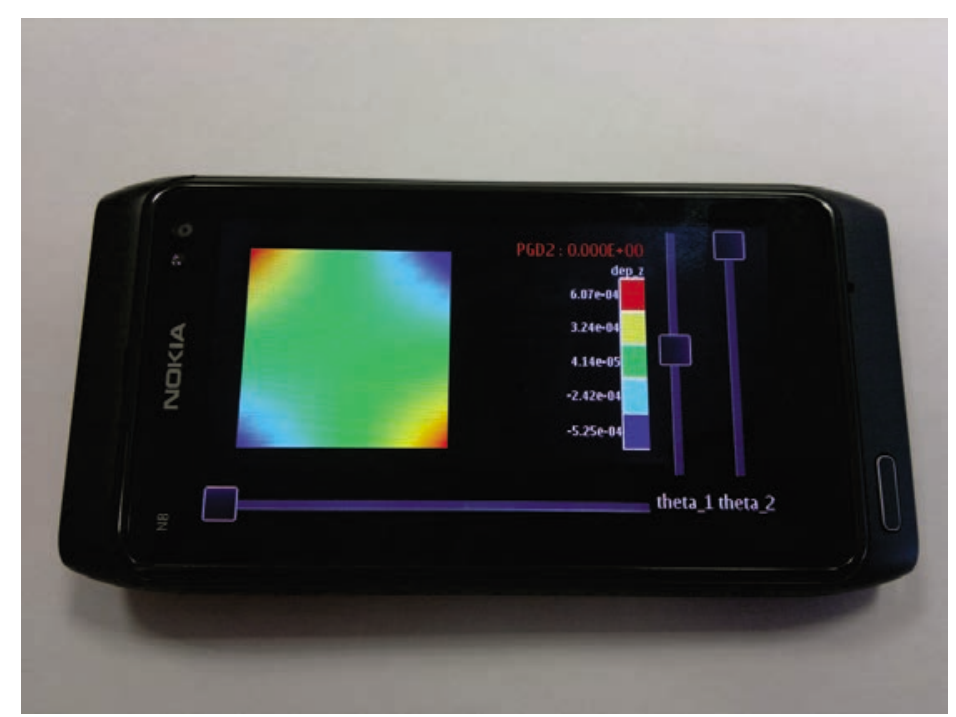

Fig. 3 Composite laminate analysis on a smartphone

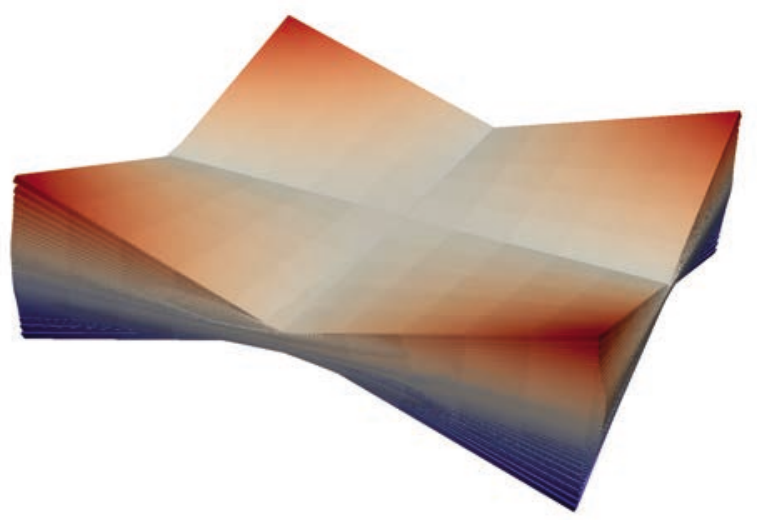

Fig. 4 Deformation envelope generated by all combinations of the reinforcement orientations of the top and bottom plies

we simulate an unbalanced laminate by acting on both vertical sliders, the plate deforms. By assuming a certain uncertainty in the real orientation of such plies, one can evaluate the envelope of the resulting distorted structures due to the thermomechanical coupling as depicted in Fig. 4.

\subsection{Inverse identification and optimization}

It is easy to understand that after performing this type of calculations, in which parameters are considered advantageously as new coordinates of the model, a posteriori inverse identification or optimization can be easily handled. This new PGD framework allows us to perform this type of calculations very efficiently, because in fact all possible solutions have been previously computed in the form of a separated, high-dimensional so- 
lution so that they constitute a simple post-processing of this general solution. Process optimization was considered in [34], for instance. Shape optimization was performed in [50] by considering all the geometrical parameters as extra-coordinates, leading to the model solution in any of the geometries generated by the parameters considered as extra-coordinates.

We consider the Laplace equation defined in the parametrized domain $\Omega^{r}$ described from 12 control points $\mathcal{P}_{i}^{r}, i=1, \cdots, 12$, with coordinates

$$
\left\{\begin{array}{l}
\mathcal{P}_{1}^{r}=(0,0) \\
\mathcal{P}_{2}^{r}=(1,0) \\
\mathcal{P}_{3}^{r}=(2,0) \\
\mathcal{P}_{4}^{r}=(3,0) \\
\mathcal{P}_{5}^{r}=(4,0) \\
\mathcal{P}_{6}^{r}=(5,0) \\
\mathcal{P}_{7}^{r}=(5,1) \\
\mathcal{P}_{8}^{r}=(4,1) \\
\mathcal{P}_{9}^{r}=(3,1) \\
\mathcal{P}_{10}^{r}=(2,1) \\
\mathcal{P}_{11}^{r}=(1,1) \\
\mathcal{P}_{12}^{r}=(0,1)
\end{array}\right.
$$

Different polygonal domains $\Omega$ are obtained by moving vertically points $\mathcal{P}_{i}^{r}, i=$ $7, \cdots, 12$, being defined by:

$$
\left\{\begin{array}{l}
\mathcal{P}_{1}=(0,0) \\
\mathcal{P}_{2}=(1,0) \\
\mathcal{P}_{3}=(2,0) \\
\mathcal{P}_{4}=(3,0) \\
\mathcal{P}_{5}=(4,0) \\
\mathcal{P}_{6}=(5,0) \\
\mathcal{P}_{7}=\left(5,1+\theta_{1}\right) \\
\mathcal{P}_{8}=\left(4,1+\theta_{2}\right) \\
\mathcal{P}_{9}=\left(3,1+\theta_{3}\right) \\
\mathcal{P}_{10}=\left(2,1+\theta_{4}\right) \\
\mathcal{P}_{11}=\left(1,1+\theta_{5}\right) \\
\mathcal{P}_{12}=\left(0,1+\theta_{6}\right)
\end{array}\right.
$$

with $\theta_{i} \in[-0.3,0.3], i=1, \cdots, 6$.

The resulting separated representation of the solution involves 70 terms

$$
\begin{gathered}
u\left(\mathbf{x}, \theta_{1}, \theta_{2}, \theta_{3}, \theta_{4}, \theta_{5}, \theta_{6}\right) \approx \\
\approx \sum_{i=1}^{70} F_{i}(\mathbf{x}) \cdot \Theta_{1 i}\left(\theta_{1}\right) \cdot \Theta_{2 i}\left(\theta_{2}\right) \cdot \Theta_{3 i}\left(\theta_{3}\right) \cdot \Theta_{4 i}\left(\theta_{4}\right) \cdot \Theta_{5 i}\left(\theta_{5}\right) \cdot \Theta_{6 i}\left(\theta_{6}\right)
\end{gathered}
$$

Figure 5 compares the particularization of the general solution (67) when considering the geometry defined by $\left(\theta_{1}, \cdots, \theta_{6}\right)=(-0.3,0.3,0.3,-0.3,0.3,0.3)$, that is $u\left(\mathbf{x}, \theta_{1}=-0.3, \theta_{2}=0.3, \theta_{3}=0.3, \theta_{4}=-0.3, \theta_{5}=0.3, \theta_{6}=0.3\right)$ with the finite element solution in such a domain. We can conclude that both solutions are in perfect agreement. It is important to notice that as the interval in which coordinates $\theta_{i}(i=1, \cdots, 6)$ are defined $[-0.3,0.3]$ were discretized by suing 13 nodes uniformly 
distributed, the separated representation (67) represents the solution for $13^{6}$ different geometries, that is, for 4.826 .809 possible domain geometries. Again, the analysis can be performed in deployed devices like smartphones or tablets, in real time.
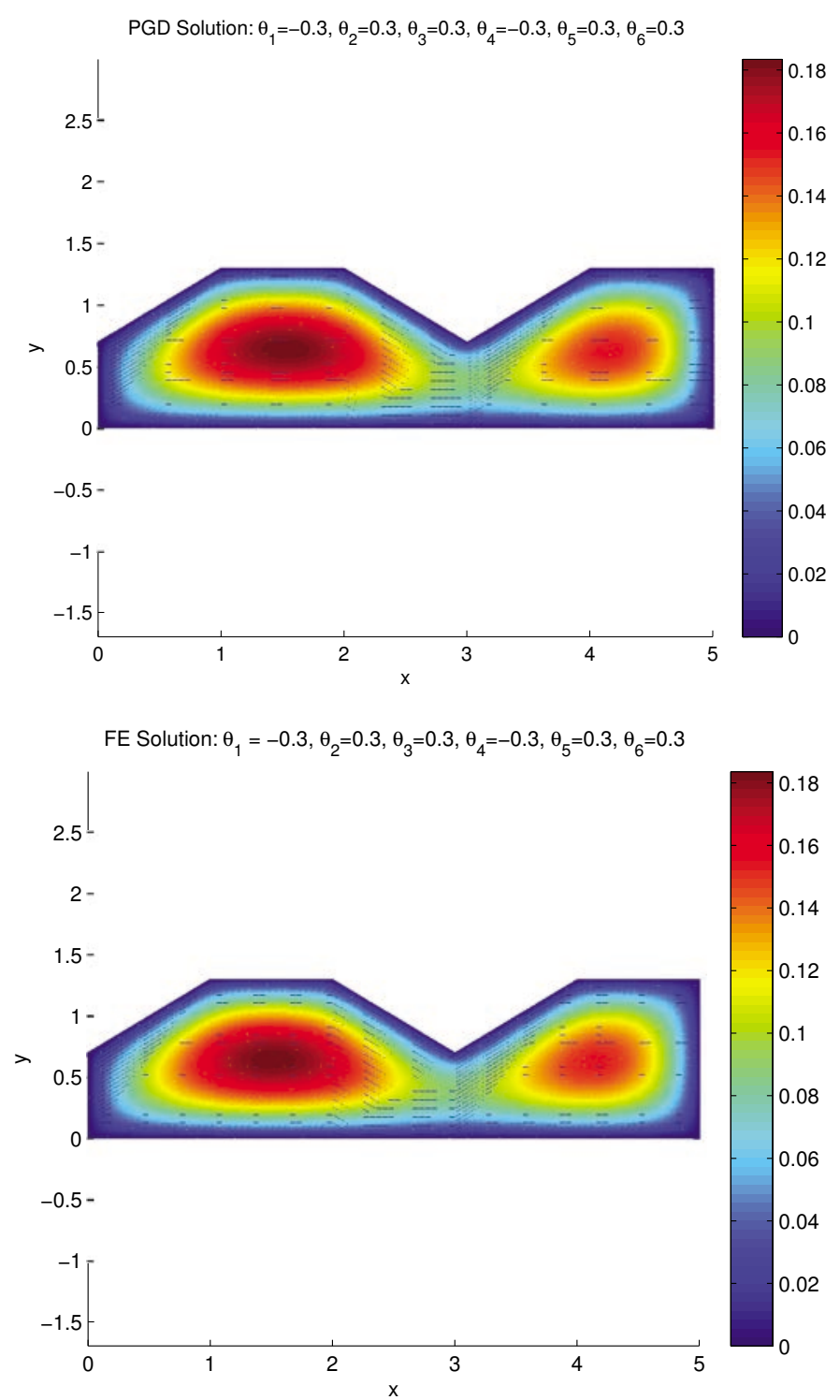

Fig. 5 Comparing $u\left(\mathbf{x}, \theta_{1}=-0.3, \theta_{2}=0.3, \theta_{3}=0.3, \theta_{4}=-0.3, \theta_{5}=0.3, \theta_{6}=0.3\right)$ with the finite element solution $u(\mathbf{x}), \mathbf{x} \in \Omega$, with $\Omega$ defined by $\theta_{1}=-0.3, \theta_{2}=0.3, \theta_{3}=0.3, \theta_{4}=$ $-0.3, \theta_{5}=0.3, \theta_{6}=0.3$.

\subsection{PGD based Dynamic Data Driven Application Systems}

Inverse methods in the context of real-time simulations were addressed in [38] and were coupled with control strategies in [35] as a first step towards DDDAS (dynamic datadriven application systems). Moreover, because the general parametric solution was pre-computed off-line, it can be used on-line under real time constraints and using light computing platforms like smartphones [21] [35], that constitutes a first step towards the use of this kind of representation in augmented reality platforms. 


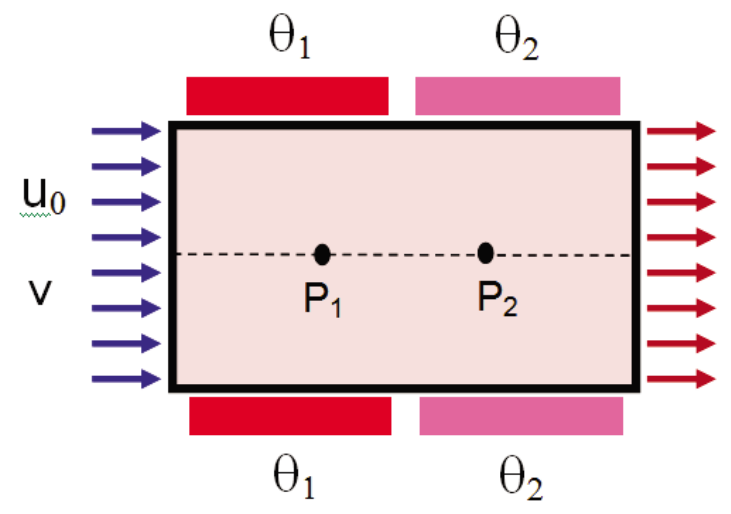

Fig. 6 Thermal process consisting of two heating devices located on the die walls where the temperature is enforced to the values $\theta_{1}$ and $\theta_{2}$ respectively.

Traditionally, Simulation-based Engineering Sciences (SBES) relied on the use of static data inputs to perform the simulations. These data could be parameters of the model(s) or boundary conditions, outputs at different time instants, etc., traditionally obtained through experiments. The word static is intended here to mean that these data could not be modified during the simulation.

A new paradigm in the field of Applied Sciences and Engineering has emerged in the last decade. Dynamic Data-Driven Application Systems (DDDAS) constitute nowadays one of the most challenging applications of SBES. By DDDAS we mean a set of techniques that allow the linkage of simulation tools with measurement devices for real-time control of simulations and applications. As defined by the U.S. National Science Foundation, "DDDAS entails the ability to dynamically incorporate additional data into an executing application, and in reverse, the ability of an application to dynamically steer the measurement process" [71].

An important issue encountered in DDDAS, related to process control and optimization, inverse analysis, etc., lies in the necessity of solving many direct problems. Thus, for example, process optimization implies the definition of a cost function and the search of optimum process parameters, which minimize the cost function. In most engineering optimization problems the solution of the model is the most expensive step. Real-time computations with zero-order optimization techniques can not be envisioned except for very particular cases. The computation of sensitivity matrices and adjoint approaches also hampers fast computations. Moreover, global minima are only ensured under severe conditions, which are not (or cannot be) verified in problems of engineering interest.

Multidimensionality offers an alternative getaway to avoid too many direct solutions. In this section the main ideas related to casting the model into a multidimensional framework, followed by process optimization, are introduced. For the sake of clarity in what follows we consider the thermal model related to a material flowing into a heated die. Despite the apparent simplicity, the strategy here described can be extended to address more complex scenarios.

The $2 \mathrm{D}$ thermal process is sketched in Figure 6 . The material flows with a velocity $\mathbf{v}$ inside a die $\Omega$ of length $L$ and width $H$. The temperature of the material at the die entrance is $u_{0}$. The die is equipped with two heating devices of lengths $L_{1}$ and $L_{2}$ respectively, whose temperatures $\theta_{1}$ and $\theta_{2}$ respectively, can range within an interval $\left[\theta_{\min }, \theta_{\max }\right]$. 
The die is equipped with two heating devices as depicted in Figure 6 whose temperatures constitute the process parameters to be optimized and, eventually, controlled. For the sake of simplicity the internal heat generation $Q$ is assumed constant, as well as the velocity $v$ and the inlet temperature $u_{0}$.

Different values of prescribed temperatures at both heating devices can be considered. The resulting 2D heat transfer equation can be then solved. As noted earlier, optimization or inverse identification will require many direct solutions or, as named in the introduction, static data computations. Obviously, when the number of the process parameters involved in the model is increased, standard approaches fail to compute optimal solutions in a reasonable time. Thus, for a large number of process parameters, real-time computations are precluded and, moreover, performing "on-line" optimization or inverse analysis is a challenging issue.

The method proposed in [35] consists on introducing both process parameters, i.e. temperatures of the heating devices, $\theta_{1}$ and $\theta_{2}$, as extra coordinates.

To circumvent the curse of dimensionality related to the high dimensional space in which the temperature field $u\left(x, y, \theta_{1}, \theta_{2}\right)$ is defined - which we retain to be fourdimensional for the ease of exposition - we consider a separated representation of that field:

$$
u\left(x, y, \theta_{1}, \theta_{2}\right) \approx \sum_{i=1}^{N} F_{i}(x, y) \Theta_{i}^{1}\left(\theta_{1}\right) \Theta_{i}^{2}\left(\theta_{2}\right)
$$

where all the functions involved in such separated representation are computed by applying the Proper Generalized Decomposition technique, described previously.

Optimization procedures look for optimal parameters minimizing an appropriate single or multi objective cost function (sometimes subjected to many constraints). In this work we consider a simple scenario, in which the cost function only involves the coldest thermal history of an imaginary material particle traversing the die, it is expressed as:

$$
\mathcal{C}\left(\theta_{1}, \theta_{2}\right)=\frac{1}{2}\left(\int_{0}^{L} u\left(x, \frac{H}{2}, \theta_{1}, \theta_{2}\right) d x-\beta\right)^{2},
$$

where $\beta$ denotes the optimal value of the thermal history able to ensure a certain material transformation. Values lower than $\beta$ imply that the material has not received the necessary amount of heat, whereas values higher than $\beta$ imply an unnecessary extra-heating.

Now, optimal process parameters $\theta_{1}^{\text {opt }}$ and $\theta_{2}^{\text {opt }}$ must be calculated by minimizing the cost function. There exist many techniques for such minimization. The interested reader can refer to any book on optimization. Many of them proceed by evaluating the gradient of the cost function and then moving on that direction. The gradient computation involves the necessity of performing first derivatives of the cost function with respect to the process parameters. Other techniques involve the calculation of second derivatives. To this end, one should calculate the derivatives of the problem solution with respect to the optimization parameters.

It is important to note that separated representations of the process parameters drastically simplifies this task because as the solution depends explicitly on the parameters its derivation is straightforward, namely,

$$
\frac{\partial u}{\partial \theta_{1}}\left(x, y, \theta_{1}, \theta_{2}\right) \approx \sum_{i=1}^{N} F_{i}(x, y) \frac{\partial \Theta_{i}^{1}}{\partial \theta_{1}}\left(\theta_{1}\right) \Theta_{i}^{2}\left(\theta_{2}\right)
$$


and

$$
\frac{\partial u}{\partial \theta_{2}}\left(x, y, \theta_{1}, \theta_{2}\right) \approx \sum_{i=1}^{N} F_{i}(x, y) \Theta_{i}^{1}\left(\theta_{1}\right) \frac{\partial \Theta_{i}^{2}}{\partial \theta_{2}}\left(\theta_{2}\right) .
$$

Note that second derivatives are also similarly obtained. The calculation of the solution derivatives is a tricky point when proceeding from standard discretization techniques because the parametric dependency of the solution is, in general, not explicit.

In the simulations carried out in what follows, the minimization of the cost function was performed by using a Levenberg-Marquardt algorithm, see [33] for further details.

By performing an inverse analysis it is also possible to determine a hypothetical malfunctioning of the system, along with the determination of the broken heater. This inverse identification can easily be done in real-time by minimizing a new cost function involving the distance of the measurements to the optimal solution obtained before. The last step consists in the reconfiguration of the system, assuming that the broken heater cannot be replaced for a while. Again, a minimization procedure of the cost function, Eq. (69), this time with one fixed temperature (that of the broken heater) serves to this purpose. An implementation of this procedure on a smartphone can be done easily, see Fig. 7.

\subsection{Surgery simulators}

As mentioned before, surgical simulators must provide feedback response frequencies higher than $500 \mathrm{~Hz}$. This means that we must solve problems involving material and geometrical nonlinearities close to one thousand times per second. It is now clear that the use of model reduction seems to be an appealing alternative for reaching such performances. However, techniques based on the use of POD, POD with interpolation (PODI), even combined with asymptotic numerical methods to avoid the computation of the tangent stiffness matrix [31] [50], exhibit serious difficulties to fulfil such requirements as discussed in [56] [57] [58] [59].

Here, parametric solutions are envisaged in which the applied load $\mathbf{p}$ and its point of application $\mathbf{y}$ are considered as extra-coordinates, allowing the off-line calculation of the parametric solution:

$$
u_{j}(\mathbf{x}, \mathbf{p}, \mathbf{y}) \approx \sum_{i=1}^{N} X_{i}^{j}(\mathbf{x}) \cdot P_{i}^{j}(\mathbf{p}) \cdot Y_{i}^{j}(\mathbf{y})
$$

Again, the obtained, off-line, solution is exploited in real time even on smartphones and tablets, see Fig. 8 for an Android implementation.

For a liver palpation simulation, for instance, model's solution was composed by a total of $N=167$ functional pairs. The third component (thus $j=3$ ) of the first six spatial modes $\mathbf{X}(\mathbf{x})$ is depicted in Fig. 9. The same is done in Fig. 10 for functions $\mathbf{Y}$, although in this case they are defined only on the boundary of the domain, i.e., $\bar{\Gamma}=\partial \Omega$.

In this case, an explicit linearization of the resulting system of equations was employed, although other more sophisticated techniques could equally be employed.

Noteworthy, both $\mathbf{X}$ and $\mathbf{Y}$ sets of functions present a structure similar to that generated by Proper Orthogonal Decompositions methods, despite the fact that they are not, in general, optimal. Note how the frequency content of each pair of functions increases as we increase the number of the function, $k$. 


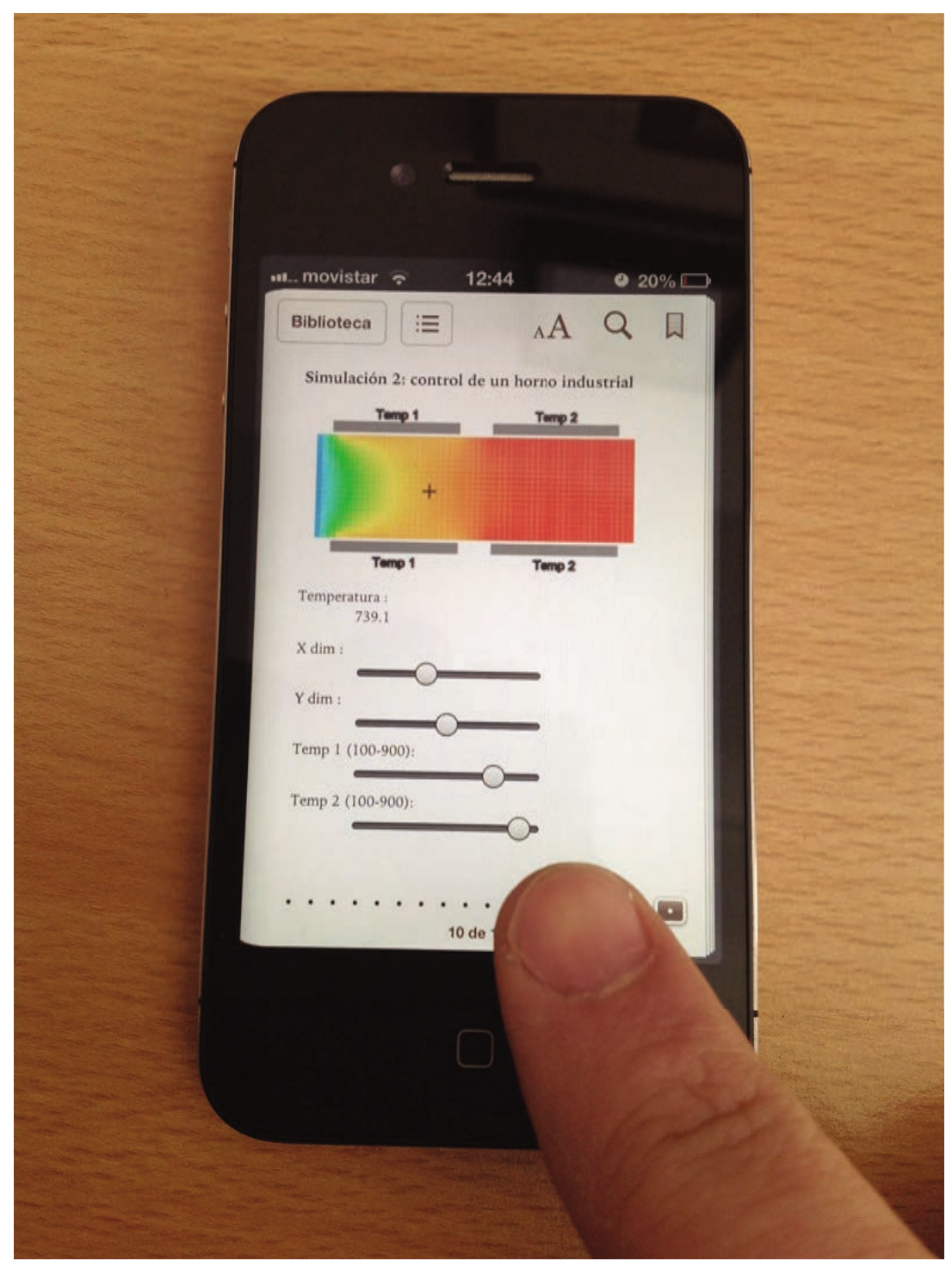

Fig. 7 Implementation of the technique described before on an iPhone. Simple formats such as the epub open format, that enables javascript, suffices implement this technique.

The solution provided by the method agrees well with reference FE solutions obtained employing full-Newton-Raphson iterative schemes (following the same tendency than that shown for the beam bending problem). But, notably, the computed solution can be stored in a so compact form that an implementation of the method is possible on handheld devices such as smartphones and tablets. For more sophisticated requirements, such as those dictated by haptic peripherals, a simple laptop (in our case a MacBook pro running MAC OSX 10.7.4, equipped with 4 Gb RAM and an Intel core i7 processor at $2.66 \mathrm{GHz}$ ) is enough to achieve this performance, see Fig. 11.

\subsection{Other industrial applications}

In [30] [65] authors addressed an industrial application for on-line simulation and material and process characterization of automated tape placement for composite forming processes. This application is at present running at the industrial level in different platforms: laptop, tablets and smartphones. Its application for training purposes is being explored, and the first accomplishments were reported in [22]. 


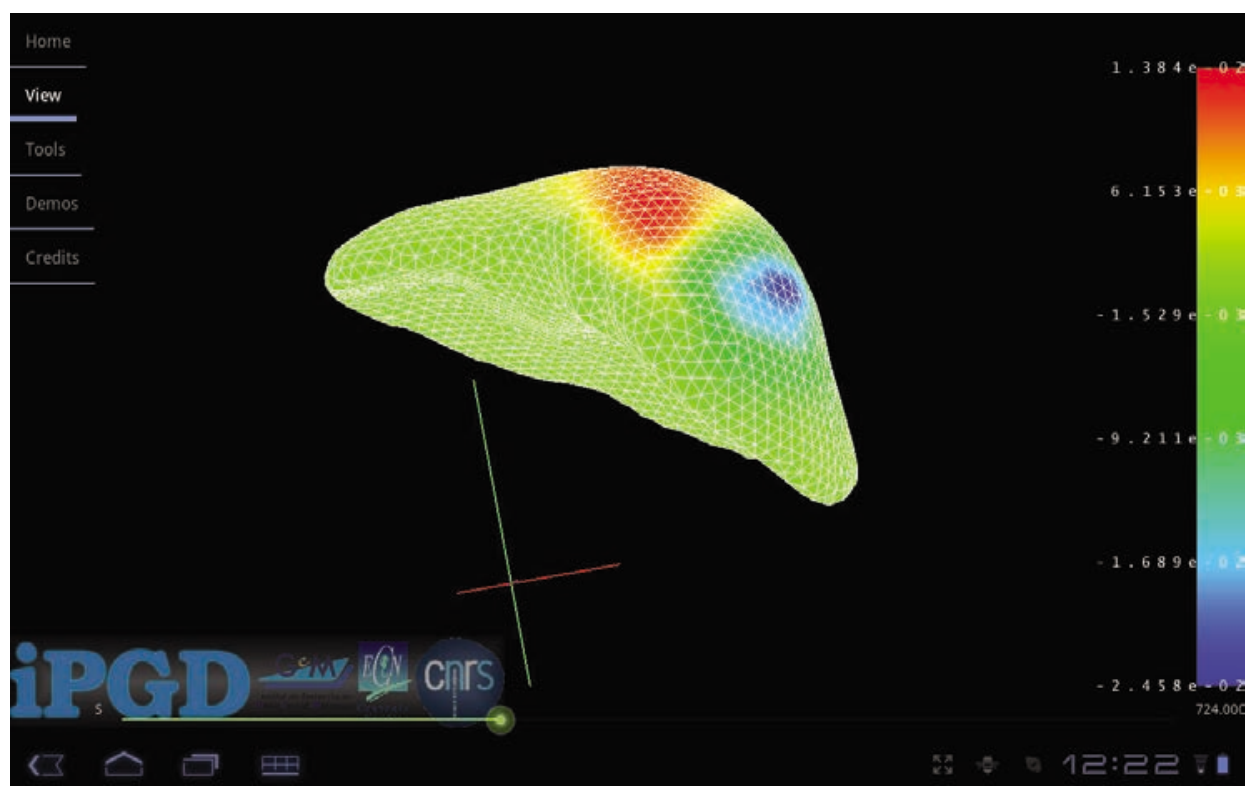

Fig. 8 Towards real time surgical simulations based on parametric PGD-based virtual charts

\section{Conclusions}

In this paper we proved that models can be enriched by introducing model parameters as extra-coordinates. Thus, one can introduce boundary conditions, material or process parameters, initial conditions, geometrical parameters, ... as extra-coordinates in order to compute general parametric solutions that define a sort of virtual charts or metamodels, much more rich that the ones obtained by sampling the parametric space. The price to be paid is the increase of the model dimensionality, but the separated representations involved in the so called PGD method allows circumventing efficiently this numerical illness. Moreover, the parametric solution is calculated in a sort of compressed format allowing for cheap storage and post-treatment. Thus, only one off-line heavy solution is needed for computing the parametric solution that constitutes the virtual chart that is then used on-line, sometimes in real time, in deployed devices as tablets or smartphones.

This off-lie/on-line approach opens numerous possibilities in the context of simulation based engineering for simulating, optimizing or controlling materials, processes and systems.

Until now, the results obtained are very encouraging, however a major difficulty persists, the one related to the solution of parametric non-linear models involving multiscale and multi-physics complex couplings. Different possibilities are being explored, as the one of combining PGD with a POD based treatment of the strong non-linearities, the use of empirical interpolations of non-linear terms, the use of advanced non-linear solvers like the LATIN method proposed many years ago by P. Ladeveze or the one based on the use of asymptotic expansions [51]...

If the next future non-linear parametric models can be addressed with the same simplicity than the linear ones, parametric PGD based virtual charts could open a new age for the XXI century design, optimization and control of materials, processes and systems, revolutioning the ICTs technologies. 


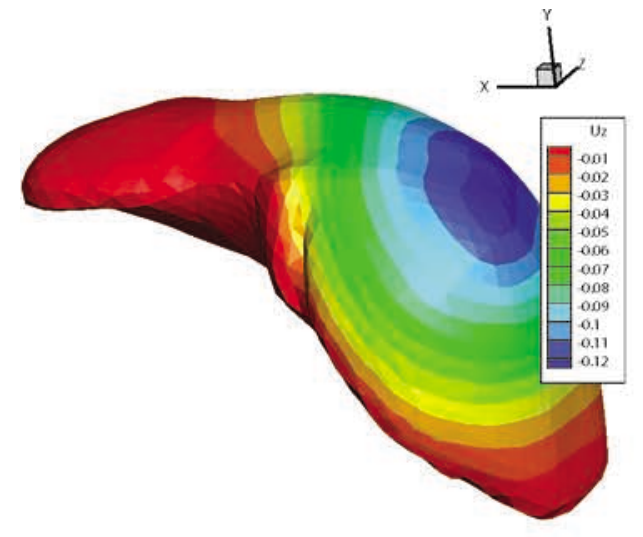

(a)

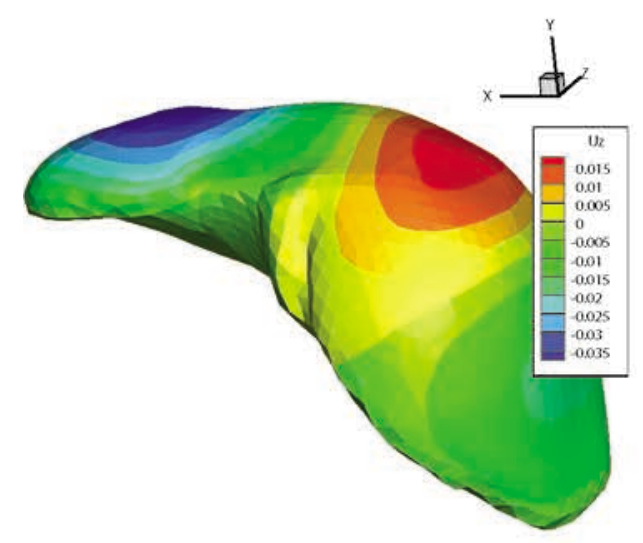

(c)

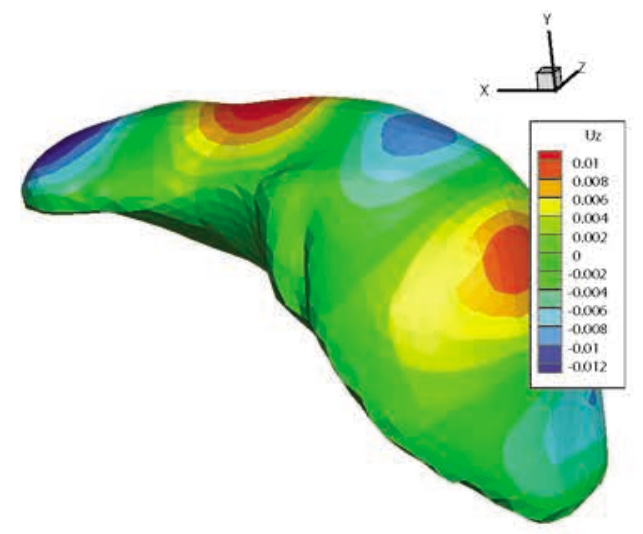

(e)

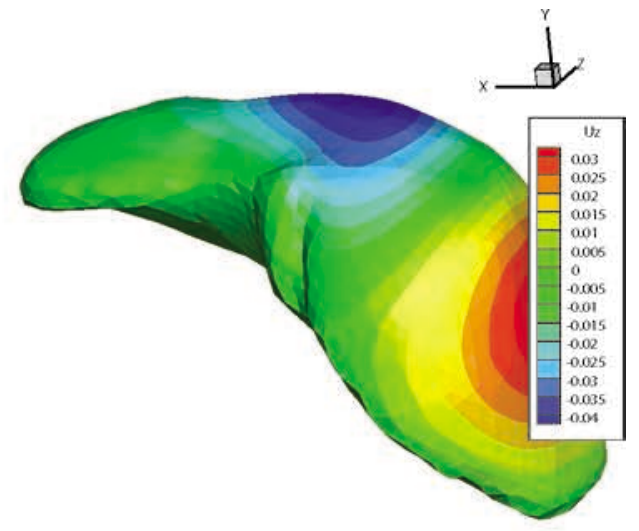

(b)

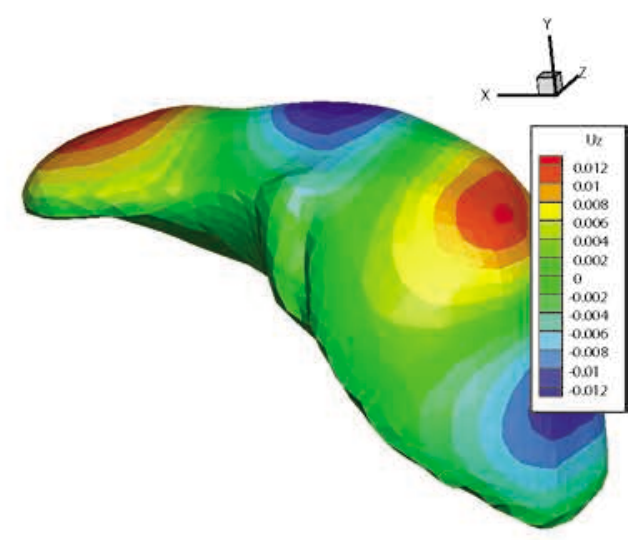

(d)

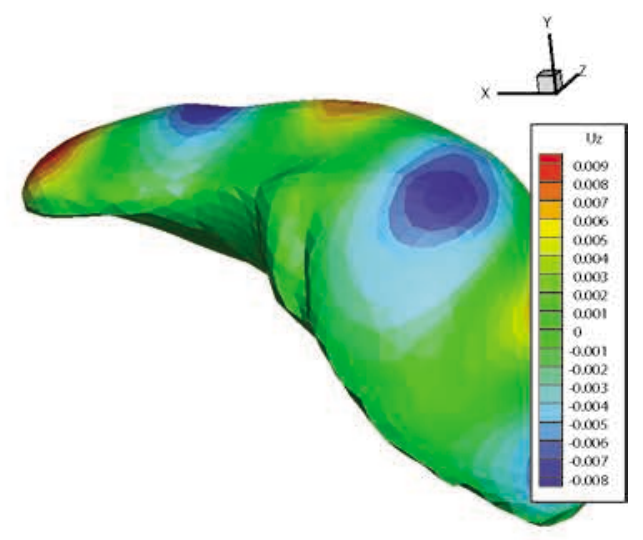

(f)

Fig. 9 Six first functions $\mathbf{X}(\mathbf{x}), k=1, \ldots 6$, for the simulation of the liver. 


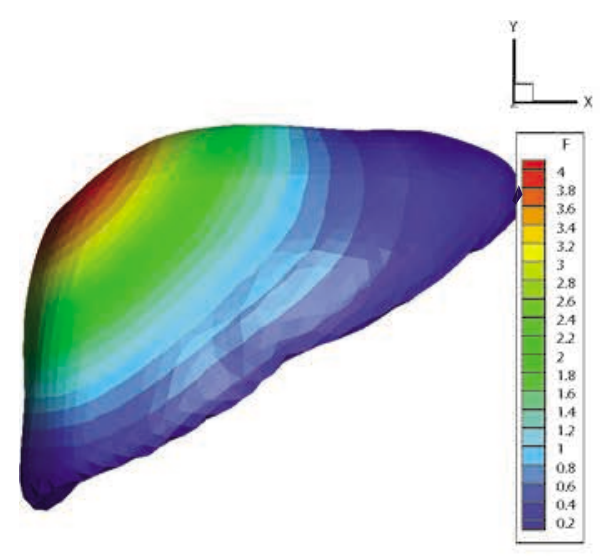

(a)

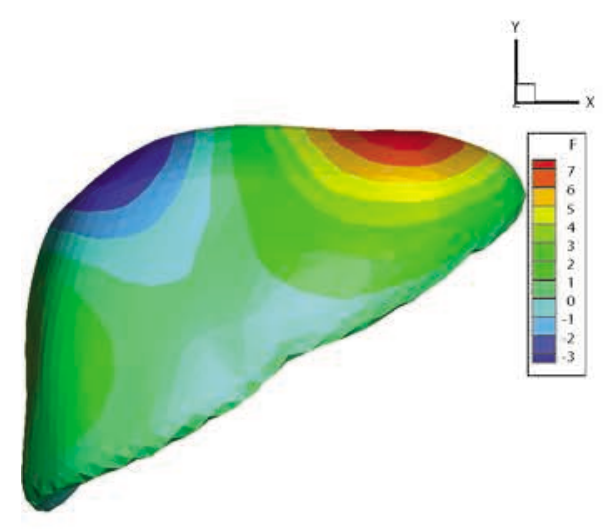

(c)

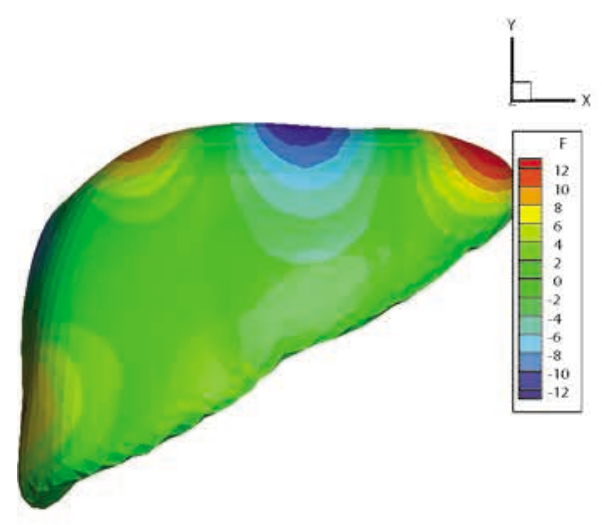

(e)

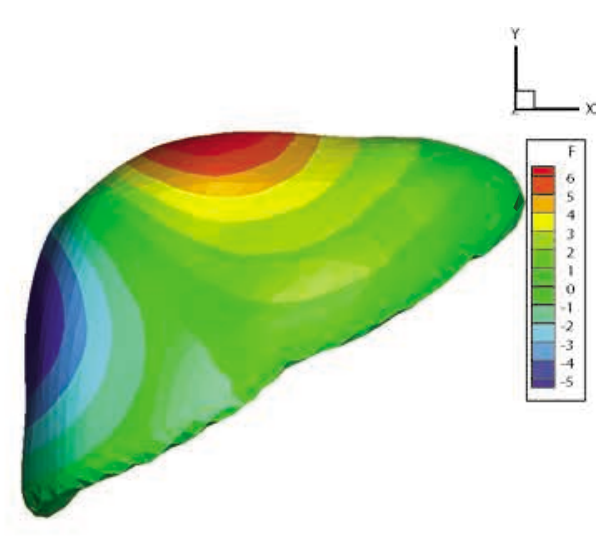

(b)

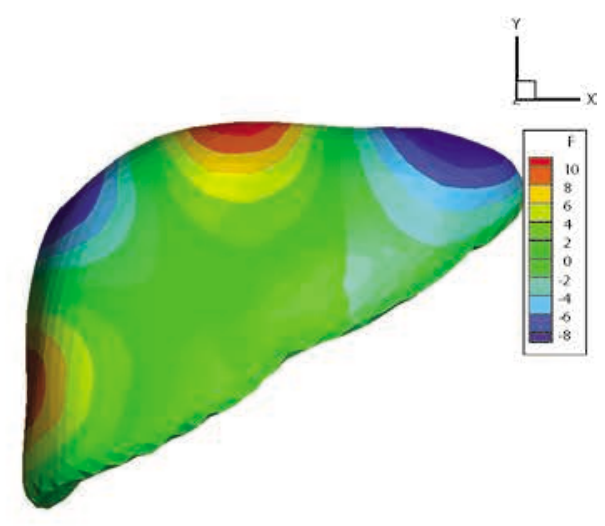

(d)

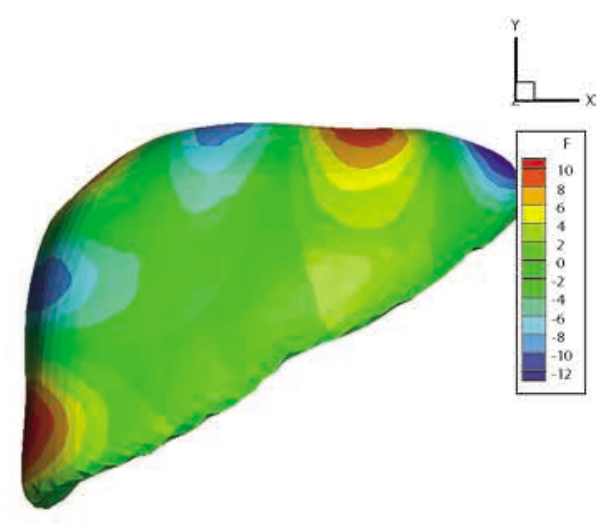

(f)

Fig. 10 Six first functions $\mathbf{Y}(\mathbf{y}), k=1, \ldots 6$, for the simulation of the liver. Note that, in this case, functions $\mathbf{Y}(\mathbf{y})$ are defined on the boundary of the liver only. 


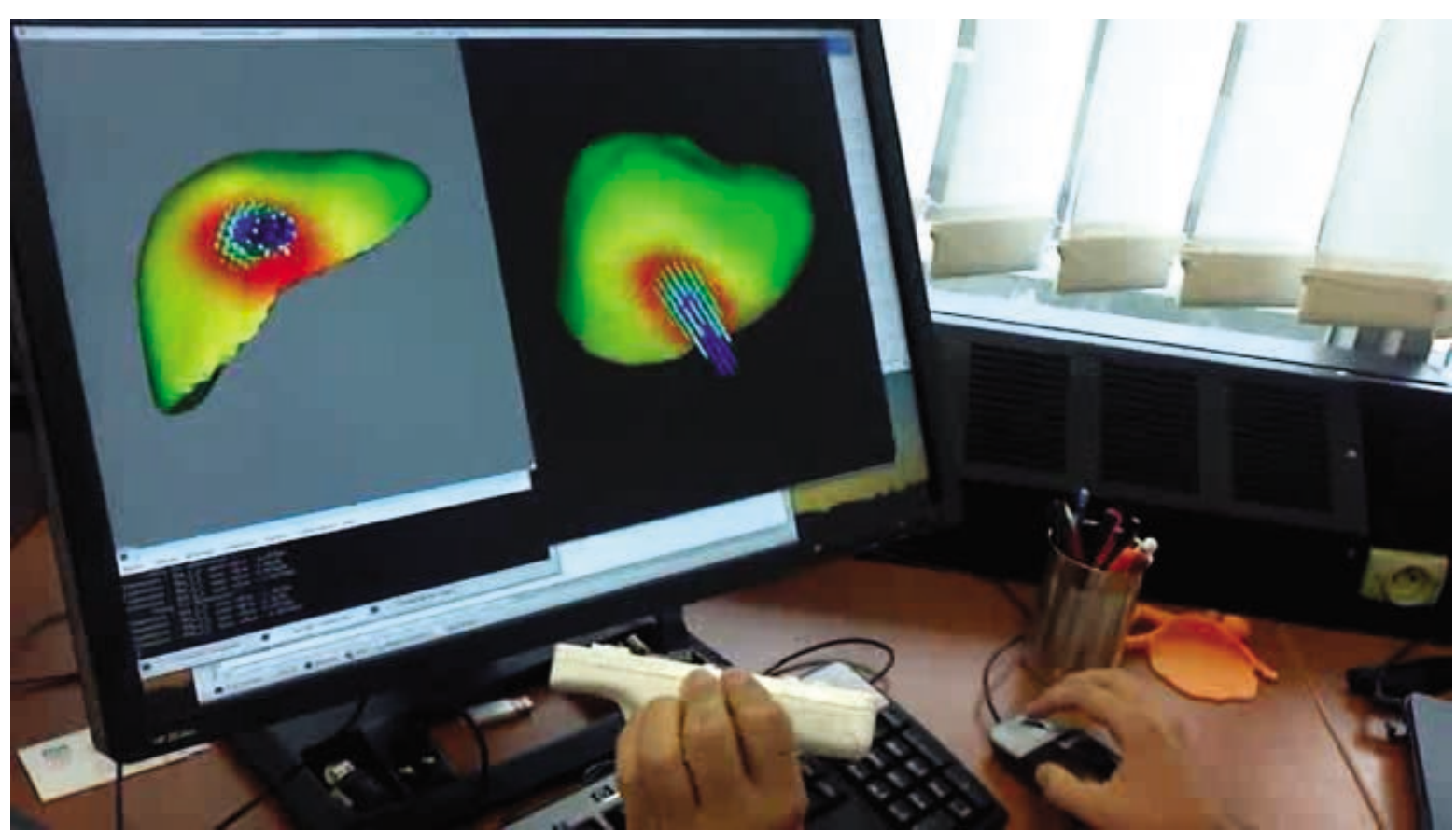

Fig. 11 Implementation of the proposed technique on a PC.

\section{A Alternating directions separated representation constructor}

A.1 Computing $R(\mathbf{x})$ from $S(t)$ and $W(k)$

We consider the extended weighted residual form of equation (8):

$$
\int_{\Omega \times \mathcal{I}_{t} \times \mathcal{I}_{k}} u^{*}\left(\frac{\partial u}{\partial t}-k \cdot \Delta u-f\right) d \mathbf{x} \cdot d t \cdot d k=0
$$

where the trial and test functions write respectively:

$$
u^{n}(\mathbf{x}, t, k)=\sum_{i=1}^{n-1} X_{i}(\mathbf{x}) \cdot T_{i}(t) \cdot K_{i}(k)+R(\mathbf{x}) \cdot S(t) \cdot W(k)
$$

and, assuming $S$ and $W$ known from the previous iteration,

$$
u^{*}(\mathbf{x}, t, k)=R^{*}(\mathbf{x}) \cdot S(t) \cdot W(k)
$$

Introducing (72) and (73) into (71) it results:

$$
\begin{aligned}
& \int_{\Omega \times \mathcal{I}_{t} \times \mathcal{I}_{k}} R^{*} \cdot S \cdot W \cdot\left(R \cdot \frac{\partial S}{\partial t} \cdot W-k \cdot \Delta R \cdot S \cdot W\right) d \mathbf{x} \cdot d t \cdot d k= \\
& =-\int_{\Omega \times \mathcal{I}_{t} \times \mathcal{I}_{k}} R^{*} \cdot S \cdot W \cdot \mathcal{R}^{n-1} d \mathbf{x} \cdot d t \cdot d k
\end{aligned}
$$

where $\mathcal{R}^{n-1}$ defines the residual related to $u^{n-1}(\mathbf{x}, t, k)$ :

$$
\mathcal{R}^{n-1}=\sum_{i=1}^{n-1} X_{i} \cdot \frac{\partial T_{i}}{\partial t} \cdot K_{i}-\sum_{i=1}^{n-1} k \cdot \Delta X_{i} \cdot T_{i} \cdot K_{i}-f
$$

Once all functions involving time and conductivity have been determined, we can integrate Eq. (74) along its respective domains $\mathcal{I}_{t} \times \mathcal{I}_{k}$, and by taking into account the following 
notations:

$$
\left[\begin{array}{rlrlrl}
w_{1} & =\int_{\mathcal{I}_{k}} W^{2} d k & s_{1} & =\int_{\mathcal{I}_{t}} S^{2} d t & r_{1} & =\int_{\Omega} R^{2} d \mathbf{x} \\
w_{2} & =\int_{\mathcal{I}_{k}} k W^{2} d k & s_{2} & =\int_{\mathcal{I}_{t}} S \cdot \frac{d S}{d t} d t & r_{2} & =\int_{\Omega} R \cdot \Delta R d \mathbf{x} \\
w_{3} & =\int_{\mathcal{I}_{k}} W d k & s_{3} & =\int_{\mathcal{I}_{t}} S d t & r_{3} & =\int_{\Omega} R d \mathbf{x} \\
w_{4}^{i} & =\int_{\mathcal{I}_{k}} W \cdot K_{i} d k & s_{4}^{i} & =\int_{\mathcal{I}_{t}} S \cdot \frac{d T_{i}}{d t} d t r_{4}^{i} & =\int_{\Omega} R \cdot \Delta X_{i} d \mathbf{x} \\
w_{5}^{i} & =\int_{\mathcal{I}_{k}} k W \cdot K_{i} d k & s_{5}^{i} & =\int_{\mathcal{I}_{t}} S \cdot T_{i} d t & r_{5}^{i} & =\int_{\Omega} R \cdot X_{i} d \mathbf{x}
\end{array}\right]
$$

Eq. (74) is reduced to:

$$
\begin{aligned}
& \int_{\Omega} R^{*} \cdot\left(w_{1} \cdot s_{2} \cdot R-w_{2} \cdot s_{1} \cdot \Delta R\right) d \mathbf{x}= \\
& =-\int_{\Omega} R^{*} \cdot\left(\sum_{i=1}^{n} w_{4}^{i} \cdot s_{4}^{i} \cdot X_{i}-\sum_{i=1}^{n} w_{5}^{i} \cdot s_{5}^{i} \cdot \Delta X_{i}-w_{3} \cdot s_{3} \cdot f\right) d \mathbf{x}
\end{aligned}
$$

Eq. (77) defines an elliptic steady-state boundary value problem that can be solved by using any discretization technique operating on the weak form of the problem (finite elements, finite volumes, ... ). Another possibility consists in coming back to the strong form of Eq. (77):

$$
\begin{gathered}
w_{1} \cdot s_{2} \cdot R-w_{2} \cdot s_{1} \cdot \Delta R= \\
=-\left(\sum_{i=1}^{n} w_{4}^{i} \cdot s_{4}^{i} \cdot X_{i}-\sum_{i=1}^{n} w_{5}^{i} \cdot s_{5}^{i} \cdot \Delta X_{i}-w_{3} \cdot s_{3} \cdot f\right)
\end{gathered}
$$

that could be solved by using any classical collocation technique (finite differences, SPH, ... ).

A.2 Computing $S(t)$ from $R(\mathbf{x})$ and $W(k)$ :

In the present case the test function is written as:

$$
u^{*}(\mathbf{x}, t, k)=S^{*}(t) \cdot R(\mathbf{x}) \cdot W(k)
$$

Now, the weighted residual form becomes:

$$
\begin{aligned}
& \int_{\Omega \times \mathcal{I}_{t} \times \mathcal{I}_{k}} S^{*} \cdot R \cdot W \cdot\left(R \cdot \frac{\partial S}{\partial t} \cdot W-k \cdot \Delta R \cdot S \cdot W\right) d \mathbf{x} \cdot d t \cdot d k= \\
& =-\int_{\Omega \times \mathcal{I}_{t} \times \mathcal{I}_{k}} S^{*} \cdot R \cdot W \cdot \mathcal{R}^{n-1} d \mathbf{x} \cdot d t \cdot d k
\end{aligned}
$$

that integrating in the space $\Omega \times \mathcal{I}_{k}$ and by taking into account the notation (76) results:

$$
\begin{aligned}
& \int_{\mathcal{I}_{t}} S^{*} \cdot\left(w_{1} \cdot r_{1} \cdot \frac{d S}{d t}-w_{2} \cdot r_{2} \cdot S\right) d t= \\
& =-\int_{\mathcal{I}_{t}} S^{*} \cdot\left(\sum_{i=1}^{n} w_{4}^{i} \cdot r_{5}^{i} \cdot \frac{d T_{i}}{d t}-\sum_{i=1}^{n} w_{5}^{i} \cdot r_{4}^{i} \cdot T_{i}-w_{3} \cdot r_{3} \cdot f\right) d t
\end{aligned}
$$

Eq. (81) represents the weak form of the ODE defining the time evolution of the field $S$ that can be solved by using any stabilized discretization technique (SU, Discontinuous Galerkin, ... ). The strong form of Eq. (81) reads:

$$
\begin{gathered}
w_{1} \cdot r_{1} \cdot \frac{d S}{d t}-w_{2} \cdot r_{2} \cdot S= \\
=-\left(\sum_{i=1}^{i=n} w_{4}^{i} \cdot r_{5}^{i} \cdot \frac{d T_{i}}{d t}-\sum_{i=1}^{i=n} w_{5}^{i} \cdot r_{4}^{i} \cdot T_{i}-w_{3} \cdot r_{3} \cdot f\right)
\end{gathered}
$$

Eq. (82) can be solved by using backward finite differences, or higher order Runge-Kutta schemes, among many other possibilities. 
Computing $W(k)$ from $R(\mathbf{x})$ and $S(t)$ :

In this part of the algorithm, the test function is written as:

$$
u^{*}(\mathbf{x}, t, k)=W^{*}(k) \cdot R(\mathbf{x}) \cdot S(t)
$$

Now, the weighted residual form becomes:

$$
\begin{aligned}
& \int_{\Omega \times \mathcal{I}_{t} \times \mathcal{I}_{k}} W^{*} \cdot R \cdot S \cdot\left(R \cdot \frac{\partial S}{\partial t} \cdot W-k \cdot \Delta R \cdot S \cdot W\right) d \mathbf{x} \cdot d t \cdot d k= \\
& =-\int_{\Omega \times \mathcal{I}_{t} \times \mathcal{I}_{k}} W^{*} \cdot R \cdot S \cdot \mathcal{R}^{n-1} d \mathbf{x} \cdot d t \cdot d k
\end{aligned}
$$

Integrating Eq. (84) in $\Omega \times \mathcal{I}_{t}$ and considering the notations given by Eq. (76) leads to:

$$
\begin{aligned}
& \int_{\mathcal{I}_{k}} W^{*} \cdot\left(r_{1} \cdot s_{2} \cdot W-r_{2} \cdot s_{1} \cdot k \cdot W\right) d k= \\
& =-\int_{\mathcal{I}_{k}} W^{*} \cdot\left(\sum_{i=1}^{n} r_{5}^{i} \cdot s_{4}^{i} \cdot K_{i}-\sum_{i=1}^{n} r_{4}^{i} \cdot s_{5}^{i} \cdot k \cdot K_{i}-r_{3} \cdot s_{3} \cdot f\right) d k
\end{aligned}
$$

Equation (85) does not involve any differential operator. The strong form of Eq. (85) is:

$$
\left(r_{1} \cdot s_{2}-r_{2} \cdot s_{1} \cdot k\right) \cdot W=-\left(\sum_{i=1}^{i=n}\left(r_{5}^{i} \cdot s_{4}^{i}-r_{4}^{i} \cdot s_{5}^{i} \cdot k\right) \cdot K_{i}-r_{3} \cdot s_{3} \cdot f\right)
$$

Eq. (86) represents an algebraic equation because the original model does not involve derivatives with respect to the conductivity. Thus, despite the introduction of parameters as additional model coordinates, the computational complexity remains essentially the same, however, the introduction of extra-coordinates implies in general the increase of the number of modes involved by the separated representation, and consequently the computing time.

\section{References}

1. http://www.epractice.eu/en/news/5304734

2. http://www.ga-project.eu/

3. http://www.humanbrainproject.eu/

4. http://www.itfom.eu/

5. http://robotcompanions.eu

6. http://www.futurict.eu

7. http://www.graphene-flagship.eu/

8. A. Ammar, B. Mokdad, F. Chinesta, R. Keunings. A new family of solvers for some classes of multidimensional partial differential equations encountered in kinetic theory modeling of complex fluids. Journal of Non-Newtonian Fluid Mechanics, 139, 153-176, 2006.

9. A. Ammar, D. Ryckelynck, F. Chinesta, R. Keunings. On the reduction of kinetic theory models related to finitely extensible dumbbells. Journal of Non-Newtonian Fluid Mechanics, 134, 136-147, 2006.

10. A. Ammar, B. Mokdad, F. Chinesta, R. Keunings. A new family of solvers for some classes of multidimensional partial differential equations encountered in kinetic theory modeling of complex fluids. Part II: Transient simulation using space-time separated representation. Journal of Non-Newtonian Fluid Mechanics, 144, 98-121, 2007.

11. A. Ammar, F. Chinesta, P. Joyot. The nanometric and micrometric scales of the structure and mechanics of materials revisited: An introduction to the challenges of fully deterministic numerical descriptions. International Journal for Multiscale Computational Engineering, 6/3, 191-213, 2008.

12. A. Ammar, E. Pruliere, F. Chinesta, M. Laso. Reduced numerical modeling of flows involving liquid-crystalline polymeres. Journal of Non-Newtonian Fluid Mechanics, 160, 140-156, 2009 . 
13. A. Ammar, F. Chinesta, A. Falco. On the convergence of a greedy rank-one update algorithm for a class of linear systems. Archives of Computational Methods in Engineering, $17 / 4,473-486,2010$.

14. A. Ammar, F. Chinesta, P. Diez, A. Huerta. An error estimator for separated representations of highly multidimensional models. Computer Methods in Applied Mechanics and Engineering, 199 1872-1880, 2010.

15. A. Ammar, M. Normandin, F. Chinesta. Solving parametric complex fluids models in rheometric flows. Journal of Non-Newtonian Fluid Mechanics, 165, 1588-1601, 2010.

16. A. Ammar, E. Cueto, F. Chinesta. Reduction of the Chemical Master Equation for gene regulatory networks using Proper Generalized Decompositions. International Journal for Numerical Methods in Biomedical Engineering, 28/9, 960-973, 2012.

17. A. Ammar, E. Cueto, F. Chinesta. Non-incremental PGD solution of parametric uncoupled models defined in evolving domains. International Journal for Numerical Methods in Engineering, In press.

18. N. Bellomo, Modeling complex living systems, Birkhauser, 2008.

19. R.A. Bialecki, A.J. Kassab, A. Fic. Proper orthogonal decomposition and modal analysis for acceleration of transient FEM thermal analysis. Int. J. Numer. Meth. Engrg., 62, 774$797,2005$.

20. B.B. Bird, C.F. Curtiss, R.C. Armstrong, O. Hassager, Dynamics of polymeric liquids, in: Kinetic Theory, vol. 2, John Wiley \& Sons, 1987.

21. B. Bognet, A. Leygue, F. Chinesta, A. Poitou, F. Bordeu. Advanced simulation of models defined in plate geometries: 3D solutions with 2D computational complexity. Computer Methods in Applied Mechanics and Engineering, 201, 1-12, 2012.

22. F. Bordeu, A. Leygue, D. Modesto, D. Gonzalez, E. Cueto, F. Chinesta. Real-time simulation techniques for augmented learning in science and engineering higher education. A PGD approach. Archives of Computational Methods in Engineering. Submitted.

23. T. Bui-Thanh, K. Willcox, O. Ghattas, B. Van Bloemen Waanders. Goal-oriented, modelconstrained optimization for reduction of large-scale systems. Journal of Computational Physics, 224/2, 880-896, 2007.

24. J. Burkardt, M. Gunzburger, H-Ch. Lee. POD and CVT-based reduced-order modeling of Navier-Stokes flows. Comput. Methods Appl. Mech. Engrg., 196, 337-355, 2006.

25. E. Cancès, M. Defranceschi, W. Kutzelnigg, C. Le Bris, Y. Maday, Computational Quantum Chemistry: a primer, Handbook of Numerical Analysis, Elsevier, Vol. X, 3-270, 2003.

26. F. Chinesta, A. Ammar, E. Cueto. Proper Generalized Decomposition of multiscale models. International Journal for Numerical Methods in Engineering, 83/8-9, 1114-1132, 2010.

27. F. Chinesta, A. Ammar, E. Cueto. Recent advances and new challenges in the use of the Proper Generalized Decomposition for solving multidimensional models. Archives of Computational Methods in Engineering, 17/4, 327-350, 2010.

28. F. Chinesta, A. Ammar, A. Leygue, R. Keunings. An overview of the Proper Generalized Decomposition with applications in computational rheology. Journal of Non Newtonian Fluid Mechanics, 166, 578-592, 2011.

29. F. Chinesta, P. Ladeveze, E. Cueto. A short review in model order reduction based on Proper Generalized Decomposition. Archives of Computational Methods in Engineering, 18, 395-404, 2011.

30. F. Chinesta, A. Leygue, B. Bognet, Ch. Ghnatios, F. Poulhaon, F. Bordeu, A. Barasinski, A. Poitou, S. Chatel, S. Maison-Le-Poec. First steps towards an advanced simulation of composites manufacturing by automated tape placement. International Journal of Material Forming. DOI 10.1007/s12289-012-1112-9

31. B. Cochelin, N. Damil, and M. Potier-Ferry. The asymptotic numerical method: an efficient perturbation technique for nonlinear structural mechanics. Revue Europeenne des Elements Finis, 3, 281-297, 1994.

32. F. Darema, Engineering/Scientific and Commercial applications: differences, similarities, and future evolution, Proceedings of the Second Hellenic European Conference on Mathematics and Informatics. HERMIS, 1, 367-374, 1994.

33. J.E. Dennis Jr. and R.B.Schnabel, Numerical methods for unconstrained optimization and nonlinear equations, Classics in Applied Mathematics, 16, Corrected reprint of the 1983 original, Society for Industrial and Applied Mathematics (SIAM), PA, 1996.

34. Ch. Ghnatios, F. Chinesta, E. Cueto, A. Leygue, P. Breitkopf, P. Villon. Methodological approach to efficient modeling and optimization of thermal processes taking place in a die: Application to pultrusion. Composites Part A, 42, 1169-1178, 2011. 
35. Ch. Ghnatios, F. Masson, A. Huerta, E. Cueto, A. Leygue, F. Chinesta. Proper Generalized Decomposition based dynamic data-driven control of thermal processes. Computer Methods in Applied Mechanics and Engineering, 213, 29-41, 2012.

36. M. Girault, E. Videcoq, D. Petit. Estimation of time-varying heat sources through inversion of a low order model built with the Modal Identification Method from in-situ temperature measurements. International Journal of Heat and Mass Transfer, 53, 206-219, 2010.

37. D. Gonzalez, A. Ammar, F. Chinesta, E. Cueto. Recent advances in the use of separated representations. International Journal for Numerical Methods in Engineering, 81/5, 637$659,2010$.

38. D. Gonzalez, F. Masson, F. Poulhaon, A. Leygue, E. Cueto, F. Chinesta. Proper Generalized Decomposition based dynamic data-driven inverse identification. Mathematics and Computers in Simulation, 82/9, 1677-1695, 2012.

39. M.D. Gunzburger, J.S. Peterson, J.N. Shadid. Reduced-order modeling of time-dependent PDEs with multiple parameters in the boundary data. Comput. Methods Appl. Mech. Engrg., 196, 1030-1047, 2007.

40. P. Ladevèze, The large time increment method for the analyze of structures with nonlinear constitutive relation described by internal variables, Comptes Rendus Académie des Sciences Paris, 309, 1095-1099, 1989.

41. P. Ladevèze, A. Nouy, A multiscale computational method with time and space homogenization, Comptes Rendus Mécanique, 330/10, 683-689, 2002.

42. P. Ladevèze, A. Nouy, O. Loiseau, A multiscale computational approach for contact problems, Computer Methods In Applied Mechanics and Engineering, 191/43, 4869-4891, 2002.

43. P. Ladevèze, A. Nouy, On a multiscale computational strategy with time and space homogenization for structural mechanics, Computer Methods In Applied Mechanics and Engineering, 192/28-30, 3061-3087, 2003.

44. P. Ladevèze, D. Néron, P. Gosselet, On a mixed and multiscale domain decomposition method, Computer Methods in Applied Mechanics and Engineering, 96, 1526-1540, 2007.

45. P. Ladevèze, J.-C. Passieux, D. Néron, The latin multiscale computational method and the proper generalized decomposition, Computer Methods In Applied Mechanics and Engineering, 199/21-22, 1287-1296, 2010.

46. P. Ladevèze, L. Chamoin, On the verification of model reduction methods based on the proper generalized decomposition, Computer Methods In Applied Mechanics and Engineering, 200, 2032-2047, 2011.

47. H. Lamari, A. Ammar, P. Cartraud, G. Legrain, F. Jacquemin, F. Chinesta. Routes for Efficient Computational Homogenization of Non-Linear Materials Using the Proper Generalized Decomposition. Archives of Computational Methods in Engineering, 17/4, 373-391, 2010.

48. H. Lamari, A. Ammar, A. Leygue, F. Chinesta. On the solution of the multidimensional Langer's equation by using the Proper Generalized Decomposition Method for modeling phase transitions. Modelling and Simulation in Materials Science and Engineering, 20/1, 015007, 2012.

49. C. Le Bris, T. Lelièvre, Y. Maday, Results and questions on a nonlinear approximation approach for solving high-dimensional partial differential equations, Constructive Approximation, 30, 621-651, 2009.

50. A. Leygue, E. Verron. A first step towards the use of Proper General Decomposition method for structural optimization. Archives of Computational Methods in Engineering, $17 / 4$, I465-472, 2010.

51. A. Leygue, F. Chinesta, M. Beringhier, T.L. Nguyen, J.C. Grandidier, F. Pasavento, B. Schrefler. Towards a framework for non-linear thermal models in shell domains. International Journal of Numerical Methods for Heat and Fluid Flow, In press.

52. Y. Maday, E.M. Ronquist. A reduced-basis element method. C. R. Acad. Sci. Paris, Ser. I, vol. 335, 195-200, 2002.

53. Y. Maday, A.T. Patera, G. Turinici. A priori convergence theory for reduced-basis approximations of single-parametric elliptic partial differential equations. Journal of Scientific Computing, 17/1-4, :437-446, 2002.

54. Y. Maday, E.M. Ronquist. The reduced basis element method: application to a thermal fin problem. SIAM J. Sci. Comput., 26/1, 240-258, 2004.

55. D. Néron, P. Ladevèze, Proper generalized decomposition for multiscale and multiphysics problems, Archives of Computational Methods In Engineering, 17/4, 351-372, 2010.

56. S. Niroomandi, I. Alfaro, E. Cueto, F. Chinesta. Real-time deformable models of non-linear tissues by model reduction techniques. Computer Methods and Programs in Biomedicine, 91, 223-231, 2008. 
57. S. Niroomandi, I. Alfaro, E. Cueto, F. Chinesta. Model order reduction for hyperelastic materials. International Journal for Numerical Methods in Engineering, 81/9, 1180-1206, 2010.

58. S. Niroomandi, I. Alfaro, E. Cueto, F. Chinesta. Accounting for large deformations in real-time simulations of soft tissues based on reduced order models. Computer Methods and Programs in Biomedicine, 105, 1-12, 2012.

59. S. Niroomandi, I. Alfaro, D. Gonzalez, E. Cueto, F. Chinesta. Real time simulation of surgery by reduced order modelling and X-FEM techniques. International Journal for Numerical Methods in Biomedical Engineering, 28/5, 574-588, 2012.

60. A. Nouy. Proper Generalized Decompositions and separated representations for the numerical solution of high dimensional stochastic problems. Archives of Computational Methods in Engineering - State of the Art Reviews, 17, 403-434, 2010.

61. NSF Final Report. DDDAS Workshop 2006, Arlington, VA, U.S.A., 2006.

62. J.T. Oden, T. Belytschko, J. Fish, T.J.R. Hughes, C. Johnson, D. Keyes, A. Laub, L. Petzold, D. Srolovitz, S.Yip, Simulation-based Engineering Science: Revolutionizing Engineering Science through simulation. NSF Blue Ribbon Panel on SBES, 2006.

63. H.M. Park, D.H. Cho. The use of the Karhunen-Loève decomposition for the modelling of distributed parameter systems. Chem. Engineer. Science, 51, 81-98, 1996.

64. J.-C. Passieux, P. Ladevèze, D.Néron, A scalable time-space multiscale domain decomposition method: Adaptive time scale separation, Computational Mechanics, 46/4, 621-633, 2010.

65. E. Pruliere, J. Ferec, F. Chinesta, A. Ammar. An efficient reduced simulation of residual stresses in composites forming processes. International Journal of Material Forming, 3/2, 1339-1350, 2010.

66. E. Pruliere, F. Chinesta, A. Ammar. On the deterministic solution of multidimensional parametric models by using the Proper Generalized Decomposition. Mathematics and Computers in Simulation, 81, 791-810, 2010.

67. G. Rozza, D.B.P. Huynh, A.T. Patera. Reduced basis approximation and a posteriori error estimation for affinely parametrized elliptic coercive partial differential equations application to transport and continuum mechanics. Archives of Computational Methods in Engineering, 15/3, 229-275, 2008.

68. D. Ryckelynck, L. Hermanns, F. Chinesta, E. Alarcon. An efficient a priori model reduction for boundary element models. Engineering Analysis with Boundary Elements, 29, 796-801, 2005.

69. D. Ryckelynck, F. Chinesta, E. Cueto, A. Ammar. On the a priori model reduction: Overview and recent developments. Archives of Computational Methods in Engineering, State of the Art Reviews, 13/1, 91-128, 2006.

70. F. Schmidt, N. Pirc, M. Mongeau, F. Chinesta. Efficient mould cooling optimization by using model reduction. International Journal of Material Forming, 4/1, 71-82, 2011.

71. Various authors, Final Report. DDDAS Workshop 2006 at Arlington, VA, U.S.A. Technical report, National Science Foundation, 2006.

72. K. Veroy, A. Patera. Certified real-time solution of the parametrized steady incompressible Navier-Stokes equations: Rigorous reduced-basis a posteriori error bounds. Int. J. Numer. Meth. Fluids, 47, 773-788, 2005.

73. E. Videcoq, O. Quemener, M. Lazard, A. Neveu. Heat source identification and on-line temperature control by a Branch Eigenmodes Reduced Model. International Journal of Heat and Mass Transfer, 51 4743-4752, 2008. 